\title{
Physical processes within a 2D granular layer during an impact
}

\author{
Franck Bourrier (1), François Nicot (1) \& Félix Darve (2)
}

(1) Cemagref - UR ETNA - 2, rue de la Papeterie - BP 76 - 38402 Saint Martin d'Hères Cedex - France (2) L3SR - INPG, UJF, CNRS - Domaine Universitaire - BP 53 -38 041 Grenoble Cedex 9 - France E-mail :franck.bourrier@cemagref.fr

\begin{abstract}
:
In this paper, the impact of a block on a coarse granular soil corresponding to rockfall events is investigated using the Discrete Element Method. Different impacting particle and medium characteristics (impact point, impacting particle size and shape, sample height, etc.) are considered.

The numerical results first exhibit the physical phenomena involved in the interaction between the impacting particle and the granular medium. The impact process starts with the partial energy exchange from the impacting particle to the soil. This phase is followed by the propagation of a shockwave from the impact point and a wave reflection on the bottom wall of the sample. A second energy exchange from soil particles to the impacting particle can occur if the reflected wave reaches the soil surface before the end of the impact.

Based on these investigations, the impacting particle bouncing occurrence diagram is defined for various impacting particle sizes, incident kinematic parameters and sample heights. The bouncing occurrence diagram brings out three impact regimes. For a small impacting particle, the impact is mainly determined by the first interaction between the impacting particle and the soil, whereas for an intermediate-sized impacting particle, the shockwave propagation through the sample is the leading phenomenon. For a large impacting particle, bouncing is associated with the formation of a compact layer below the impacting particle.
\end{abstract}

Keywords: impact, granular material, discrete element method, force chains, rockfall, shockwave

\section{Introduction}

Rockfalls are a major risk in mountainous zones since they cause serious damage to residential areas and infrastructures. Trajectory analysis of falling rocks combined with complementary geomechanical investigations is a powerful method to predict the risk encountered. Computing the falling rock trajectory comprises three stages: the separation of rock from the cliff phase, the flight phase and the bouncing phase. Many scientific problems related to modelling the separation mechanisms and the bouncing phase remain to be solved. The study presented in this paper will focus on modelling the bouncing phase in the case of the interaction of an impacting particle with a granular soil. 
In this situation, the impact process is closely related to the local configuration of soil particles near the impact point and the mechanical and geometrical properties of the impacting particle and the soil particles.

Trajectory analysis software is mainly based on global approaches to the interaction between the impacting particle and the soil. Most of currently used interaction laws (Dorren, 2003; Guzzetti et al., 2002) are built either from previous events and experimental analysis or from theoretical models (Falcetta, 1985; Descoeudres and Zimmermann, 1987; Bozzolo et al., 1988; Pfeiffer and Bowen, 1989; Kobayashi et al., 1990). The main limitation of the usual models is that they do not really account for the nature of the impact process resulting from local phenomena (Laouafa and Nicot, 2003).

In the interaction of a boulder with a granular material, the impact phase depends substantially on the ratio of the impacting block radius $R_{b}$ to the mean radius of scree particles $R_{b} / R_{m}$ (Azzoni et al., 1991). The interaction phenomenon is well established for incidents of a block impacting a fine scree slope ( $R_{b} / R_{m}>>1$ ) or a rocky ground $\left(R_{b} / R_{m}<<1\right.$ ), but a complete study is still needed to investigate the impact of a boulder on a coarse scree slope $\left(R_{b} / R_{m} \sim 1\right)$.

This configuration corresponds to the general case of the impact of a particle on a granular matter composed of particles that are the same size as the impacting particle. This phenomenon has already been investigated in order to model granular material transport processes in the fields of aeolian sand transport or particle transportation for industrial purposes, for example. In these contexts, the impact phenomenon on a monodisperse or a slightly polydisperse granular medium has already been studied both numerically and experimentally for two-dimensional (Rioual et al., 2000; Rioual et al., 2003; Ciamarra et al. 2004) and three-dimensional media (Oger et al., 2005; Beladjine et al., 2005; Beladjine et al., 2007; Tanaka et al., 2002; Nishida et al., 2004; Toiya et al., 2007). Although the impact conditions explored in these studies are different from rockfall events in terms of impacting particle size and incident kinematic parameters, the results obtained provide useful information for the interpretation of the physical phenomena related to rockfall.

The purpose of this paper is to investigate the physical mechanisms that govern the bouncing of an impacting particle onto a granular assembly. First, numerical simulations using discrete modelling of the soil are presented. Then energy exchanges between the block and the soil and energy propagation processes inside the sample are discussed. In addition, the analysis of the physical phenomena involved during impacting particle bouncing provides a definition of different impact types associated with different physical processes that depend on impacting particle size, incident kinematic parameters and soil sample properties. 


\section{A discrete modelling of the soil}

\section{1. $D E M$}

All simulations were done using the Particle Flow Code 2D software (Itasca, 1999), which is based on a DEM method (Cundall and Strack, 1979), to model two-dimensional granular material behaviour.

The DEM method used assumes that the soil is considered a collection of rigid locally deformable bodies. The interactions between particles are described by force-displacement contact laws. The first step of the solving scheme lists particle locations. Interaction forces between particles are then determined in accordance with particle locations. The particles' translational and rotational velocities are calculated from forces acting on each particle: an explicit algorithm is applied to solve Newton's second law. Finally, these velocities are used to determine new particle locations for the next time step.

The contact law governing the interactions between particles (Cundall, 1988) is based on an approximation of the Hertz-Mindlin theory (Mindlin and Deresiewicz, 1953). In this case, the forcedisplacement model is a nonlinear hypoelastic relation.
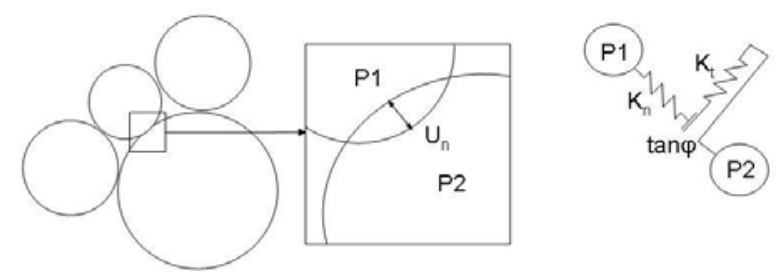

Figure 1. Contact law.

The contact law formulation (Figure 1) relates both the normal and tangential incremental contact forces $d F_{n}$ and $d F_{t}$ to the normal and the tangential incremental interpenetration $d u_{n}$ and $d u_{t}$ using the relations:

$$
\begin{aligned}
& d F_{n}=\frac{3}{2} K_{n} \sqrt{u_{n}} d u_{n} \\
& d F_{t}=K_{t} d u_{t} \xi\left(F_{n} \tan \varphi-F_{t}\right)
\end{aligned}
$$

with:

$$
\begin{aligned}
& K_{n}=\frac{2 \sqrt{2 R_{p}} G}{3(1-v)} \\
& K_{t}=2 \frac{\left(3 G^{2}(1-v) R_{p}\right)^{1 / 3}}{2-v} F_{n}^{1 / 3}
\end{aligned}
$$


where $\varphi$ is the local friction angle and $K_{n}$ and $K_{t}$ are normal and tangential stiffness coefficients depending on the mean radius of the particles involved $R_{p}$, shear modulus $G$ and Poisson's ratio $v$. $\xi$ is the Heaviside function: $\xi(x)=1$ if $x>0$ and $\xi(x)=0$ if $x \leq 0$.

The minimum time step to ensure relevant simulations is closely related to the contact force model. Indeed, the minimum time step is determined so that, during the impact simulation, any contact could be covered by a large enough number of time steps. The minimum time step is therefore dependent on the parameters of the contact model (shear modulus $G$ and Poisson's ratio $v$ ), the density of particles, the minimum radius of the spheres composing the particles and the relative velocities of the particles in contact. In the context of these simulations, the maximum relative velocities of particles during simulations allow using large time steps. The minimum time step is therefore calculated for each calculation step depending on all the other parameters cited above (Itasca, 1999), leading to time steps that are always smaller than $10^{-6} s$ during the simulations.

It can be noted that the contact force model only includes energy dissipation through frictional processes and does not introduce energy dissipation associated with viscous or plastic processes along the normal direction. Indeed, numerical simulations of the impact of a sphere on a granular packing (Oger et al., 2005) have shown that energy dissipation on the normal contact force only slightly influences the rebound of the incident particle if the particle radius is approximately the same as the mean size of the soil particles. As the main purpose of the study presented in this paper was furthering the understanding of the physical processes associated with the impact in this example $\left(R_{b} / R_{m} \sim 1\right)$, the choice was made to limit energy dissipation to frictional dissipations on the tangential contact force.

For boulder sizes larger than the mean size of the soil particles, viscous or plastic dissipation may play a role. However, in the context of the dynamical interaction of rocks, calibrating a more complex contact model based on experimental results is a very difficult task. In addition, one must keep in mind that relevant numerical modelling of energy dissipation in real systems has not yet been established conclusively and that no clear conclusions exist on the contact model to be used in discrete element methods, especially for dynamical loadings. In particular, classical discrete numerical models of granular material do not account for contact attrition, particle breakage, heat-energy dissipation and energy dissipation due to high frequency energy transfers associated with wave lengths smaller than the grain size. The assumption usually made when simulating granular assemblies with the discrete element 
method is therefore that the energy transfers and dissipation associated with particle rearrangement are predominant compared to other dissipation processes.

\subsection{Soil sample modelling}

The rock properties, size distribution and spatial settlement variability of natural scree can vary considerably depending on rockfall events. The soil characteristics are therefore fixed to the most current values encountered during rockfall events (Azzoni et al., 1991).

The impacting particle diameter varies from $0.2 \mathrm{~m}$ to $8 \mathrm{~m}$, which is in accordance with the usual range for the impacting rock size in the context of rockfall events (Kobayashi et al., 1990; Guzzetti et al., 2002). The mean size of the soil particles is set at $0.65 \mathrm{~m}$. This value allows exploring a wide range of impacting particle sizes around the mean particle size.

The soil size distribution defined accounts for the polydispersity of natural scree (Kirkby and Statham, 1975). The ratio of the volume of the largest particles to that of the smallest ones is set at 10 . The smallest particle size is limited to prevent the calculation time step from increasing.

Two typical soil models are defined to account for the influence of particle shape. The first soil type is composed of spherical particles. This soil type is hereafter called Type A (Figure 2a). The second one is a collection of clumps (Figure 2b) and will be called Type B. Clumps are indivisible assemblies of spherical particles that allow modelling the shape of soil rocks in a more realistic way. Clump assemblies can be used to model the response of a granular medium composed of complex-shaped particles to either static (Deluzarche and Cambou, 2006) or dynamic loadings (Bertrand et al., 2006). Soil type B clump assemblies are quasi-rectangular. The aspect ratio, that is, the ratio of particle length to height, is randomly chosen between 1 and 4 to account for shape variability within a talus slope. The two soil types defined have approximately the same grading curves (Figure 3). For both particle types, the density of the particles (sphere or clump assembly) is set at $2650 \mathrm{~kg} / \mathrm{m}^{3}$. 


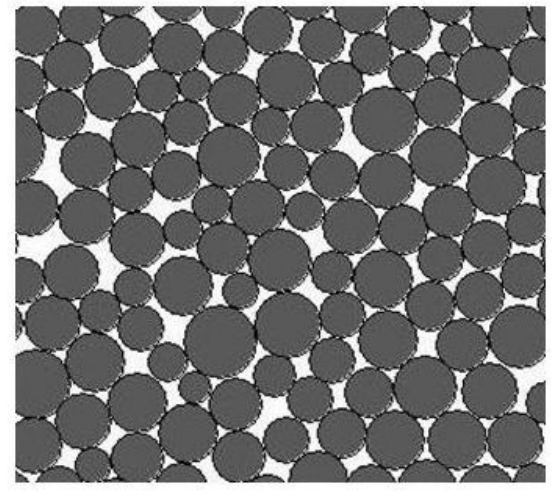

a) Spherical particles

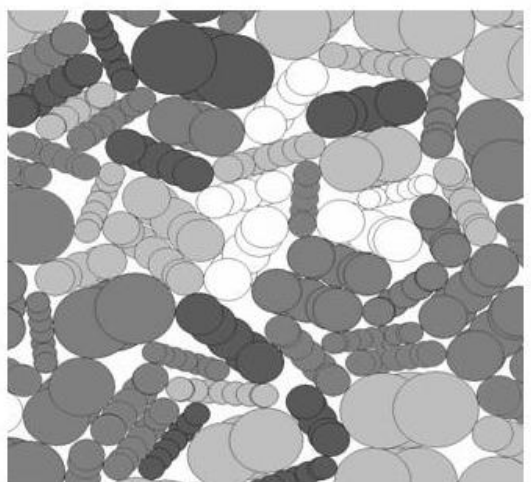

b) Clump particles

Figure 2. Particle types considered during simulations.

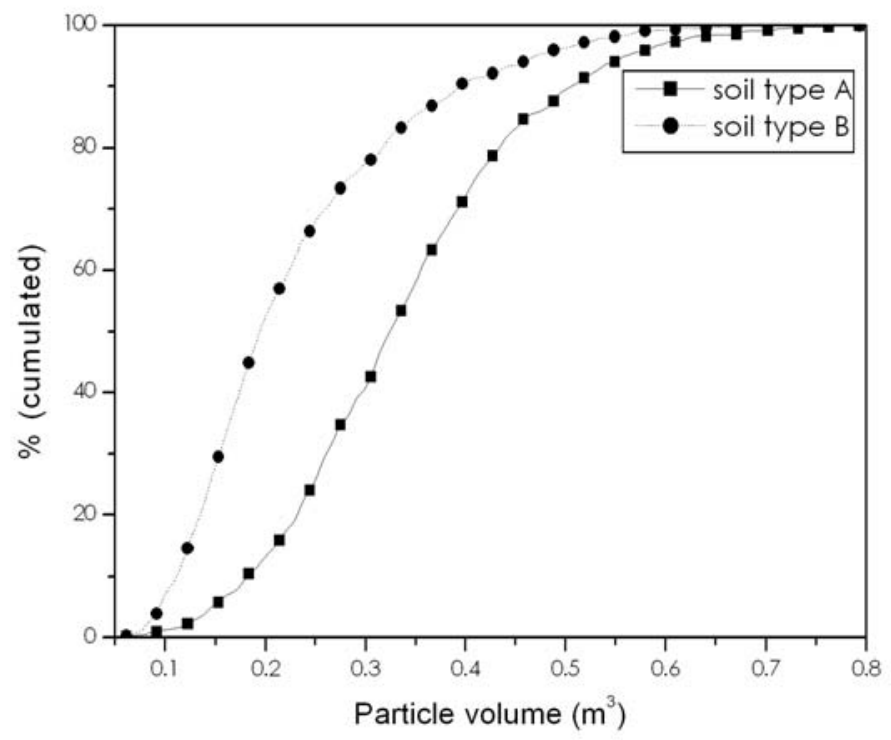

Figure 3. Soil grading curves.

Constitutive parameters are fixed in accordance with the usual values for rocks found in the literature (Goodman, 1980): the shear modulus $G$ is set at $40 \mathrm{GPa}$; Poisson's ratio $v$ is 0.25 and the local friction angle $\varphi$ is $30^{\circ}$.

The numerical soil sample is generated using the pluviation method. Among the numerous methods developed to generate granular materials using the DEM (see for example Bagi, 2005), the pluviation method is in accordance with gravitational processes leading to scree generation. This method generates a granular sample from distinct particles subjected to gravitational forces only. First the particles are 
generated within the sample. Their initial locations are determined to prevent initial interactions. Each particle length and aspect ratio is randomly defined in accordance with the material's size distribution. Gravitational forces are applied to all particles and a calculation procedure is run until the total kinetic energy of the system lowers a set value. The final porosity of the sample generated is related to the generation process. The value obtained is 0.22 for soil type A (spherical particles) and 0.2 for soil type B (clump particles).

The boundaries of the sample are fully defined by three rigid walls: two lateral walls and a bottom wall (Figure 4). The contact forces between the limiting walls and the soil particles are calculated considering each limiting wall as a discrete element with an infinite radius. The influence of the sample's height $H$ and length $2 L$ on the results of the simulations is investigated to determine the size of the soil sample (Figure 4). The length and height of the soil sample are chosen in order to remove bottom and lateral wall disturbances on the impacting particle’s bouncing.

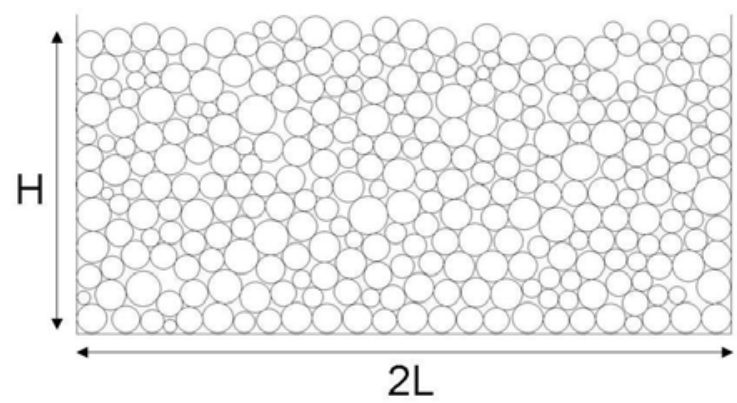

Figure 4. Soil sample.

A specific sample generation procedure is required if one wishes to use the same particle arrangement whatever the sample height and to study the influence of sample size for exactly the same soil particle spatial configuration near the impact point. The particle layout and soil surface are first defined by generating a sample with the largest size required for this specific study. The sample generated is used for all sample heights. The sample height parameter is fixed at the beginning of each simulation. In addition, a fictitious bottom wall located at $H$ value from the soil surface is created by setting to zero the displacements of all particles located below this fictitious bottom wall during all the impact simulations (Figure 5). The top layer of fixed particles models the bottom wall. The same methodology is used to study the influence of the sample length. 


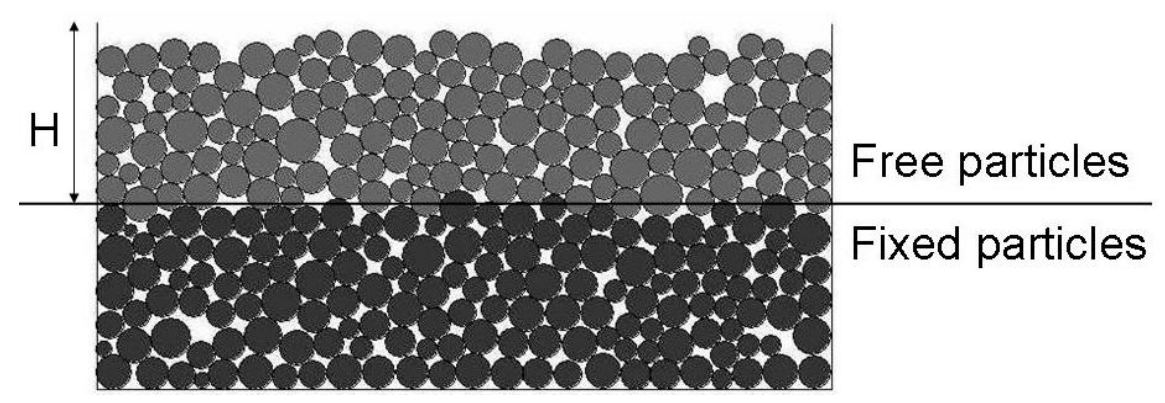

Figure 5. Sample height variation method.

Simulations for fixed impact conditions (in particular, $R_{b}=R_{m}$ ) and varying sample size show that a minimum sample size is necessary to consider the soil as infinite and eliminate wall disturbances. If the walls are located at least $9 R_{m}$ from the impact point, the walls' effects are negligible whatever the incident conditions (Figure 6). Although a smaller value would be sufficient, the length of the sample is then fixed to $2 L=50 R_{m}$ so that several impact points can be simulated on the same sample. In addition, the distance between the impact point and the lateral walls is larger than $9 R_{m}$ (see section 2.3, Figure 9), which to a large extent removes the effects of the lateral walls if $R_{b}=R_{m}$. The maximum sample height is set at $H=25 R_{m}$, which is also sufficient to simulate impacts on infinite soils if $R_{b}=R_{m}$ (Figure 6). The number of particles (spheres or clump assemblies) for the size of the sample defined above is approximately 300 .

In addition, preliminary simulations highlight that the bouncing of the boulder depends to a large extent on the height of the sample. The influence of the sample height will therefore be investigated: the sample height will be considered as a parameter that can vary up to $25 R_{m}$.

On the contrary, the influence of the lateral walls must remain negligible for all boulder sizes. For small boulders, this criterion is fulfilled since the minimum distance between the impact points and the lateral walls is chosen to remove the influence of lateral walls for $R_{b}=R_{m}$.

For larger boulders, the influence of the lateral walls might not be negligible, but completely eliminating the effect of lateral boundaries would lead to an unacceptable increase in the sample size and, as a result, of the computational time. However, as established in section 4.1 (Figure 33), bouncing is observed for sample heights that are smaller than the distances between the impact point and the lateral walls. Therefore, the effect of the lateral walls remains small compared to the influence of the sample height. 


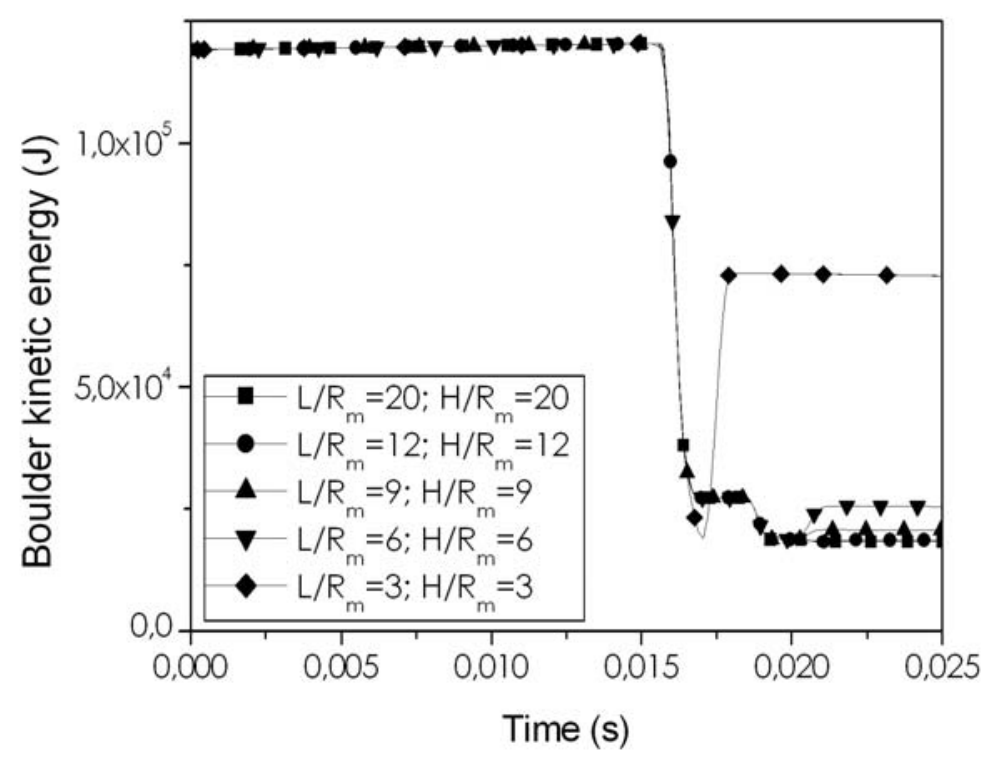

Figure 6. Influence of the sample size on the boulder's kinetic energy as it evolves during impact (beginning of the impact occurs at time $0.015 \mathrm{~s}$ ) for $R_{b}=R_{m}$.

\subsection{Impact modelling}

When generated, each soil sample is saved to be used for several impact simulations. For each impact simulation, one of the previously generated soil samples is first loaded. Incident velocities are then applied to the impacting particle at time $T_{0}$ corresponding to the beginning of the simulation. The impacting particle's horizontal location at time $T_{0}$ is fixed. Its vertical coordinate is calculated so that the first soil/impacting particle interaction occurs at time $T_{0}$.

We consider that bouncing occurs during a simulation when the impacting particle's normal velocity first vanishes followed by a sign change.

When bouncing exists, the reflected velocities (Figure 7) are collected until the time when the normal component of the impacting particle's velocity reaches its maximum (time $T_{i}$ ). This event usually occurs after the last interaction between the impacting particle and the soil particles. The impacting particle is then only subjected to gravity and its velocity decreases to reach zero for time $T_{f}$. The simulation is stopped at time $T_{f}$ so that only one impact is considered over a given simulation. 


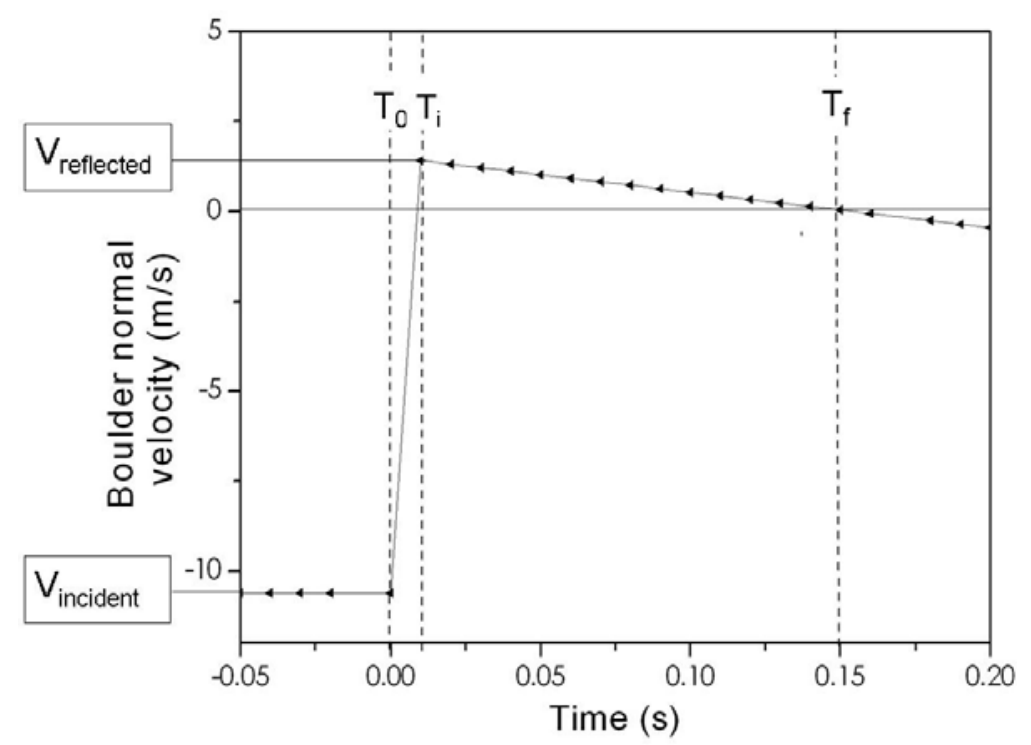

Figure 7. Definition of incident and reflected velocities.

The initial kinematic parameters of the impacting particle whose mass centre is denoted ' $G_{b}$ ' (Figure 8) are fully defined by the magnitude of the incident velocity $V_{i}$, the incident angle $\alpha_{i}$ and the incident rotational velocity $\omega_{i}$. These parameters are directly related to the normal and tangential velocity components by the relations:

$$
\begin{aligned}
& V_{n i}=V_{i} \cos \left(\alpha_{i}\right) \\
& V_{t i}=V_{i} \sin \left(\alpha_{i}\right)
\end{aligned}
$$

The incident conditions explored the range within the limits defined from rockfall events (Azzoni et al. 1991). Incident velocity $V_{i}$ ranges from $5 \mathrm{~m} / \mathrm{s}$ to $40 \mathrm{~m} / \mathrm{s}$ and the domain of the incidence angle $\alpha_{i}$ is defined as $0^{\circ}-75^{\circ}$. In this study, the incident rotational velocity is set at $\omega_{i}=0 \mathrm{rad} / \mathrm{s}$ in order to limit varying parameters.

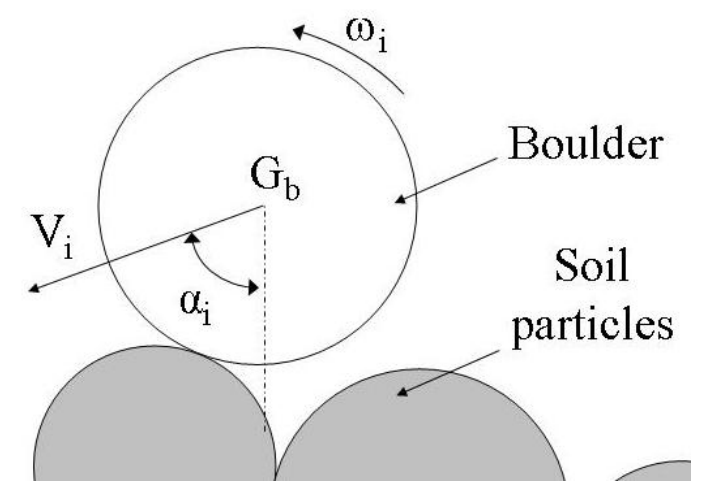


Figure 8. Incident kinematic conditions.

All energy types involved in the impact process were calculated so that energy conservation could be checked for each time step. Moreover, the changes in the different energies during the impact phase must be known so as to study the energy transfer between the impacting particle and the soil and the energy propagation within the sample.

The energies involved during the impact phase are the boulder's kinetic energy $E_{k, b}$, the total kinetic energy of all the soil particles $E_{k, s}$, the total elastic strain energy $E_{s, t}$, the total dissipated energy $E_{d, t}$ and the total gravitational potential energy $E_{p, t}$. At a given time $t$, the total elastic strain (resp. dissipated) energy corresponds to the sum of the elastic strain (resp. dissipated) energy on each contact, extended to all the contacts existing within the assembly at time $t$. The sum of all energies cited above is equal to a constant value $A$, whatever the time step considered during impact simulation:

$E_{k, b}+E_{k, s}+E_{s, t}+E_{d, t}+E_{p, t}=A$

The kinetic energy of the block $E_{k, b}$ calculated for each time step is:

$E_{k, b}=\frac{1}{2} M_{b} V_{b}^{2}+\frac{1}{2} I_{b} \omega_{b}^{2}$

where $M_{b}$ is the block mass, $I_{b}$ is the block inertia, and $V_{b}$ and $\omega_{b}$ are the block translational and rotational velocities, respectively.

The total kinetic energy of all soil particles $E_{k, s}$, the total elastic strain energy $E_{s, t}$, the total dissipated energy $E_{d, t}$ and the total gravitational potential energy $E_{p, t}$ are determined using general expressions for the calculation of the energy considered within a given domain $D$. All energies within a given domain $D$ composed of $N_{b}(t)$ particles at time $t$ are calculated using the following relations.

The total gravitational potential energy $E_{p}(D)$, which is the sum of the gravitational potential energies of all the particles within the domain $D$, is:

$E_{p}(D)=\sum_{j=1}^{N_{b}(t)}\left(M_{j} g y_{j}(t)\right)$

where $M_{j}$ is the mass of the $\mathrm{j}^{\text {th }}$ particle of the domain, $g$ is the gravitational acceleration and $y_{j}(t)$ is the vertical coordinate of the particle ' $j$ ' at time $t$. The origin of the axis of vertical coordinates corresponds to the soil surface at the beginning of the simulation. 
In the context of the simulations performed in this study, the fluctuations of the gravitational potential energy of the particles are negligible since the particles' vertical displacements during the impact phase are small.

The total kinetic energy $E_{k}(D)$, which is the sum of translational and rotational kinetic energies of all the particles within the domain $D$, is:

$$
E_{k}(D)=\sum_{j=1}^{N_{b}(t)}\left(\frac{1}{2} M_{j} V_{j}^{2}(t)+\frac{1}{2} I_{j} \omega_{j}^{2}(t)\right)
$$

where $I_{j}$ is the inertia of the $\mathrm{j}^{\text {th }}$ particle of the domain, $V_{j}(t)$ and $\omega_{j}(t)$ are the translational and rotational velocities of the particle ' $j$ ' at time $t$.

Assuming that the tangential displacement increment $d u_{t, j}(t)$ can be broken down into an elastic component $d u_{t, j}^{e}(t)$ and a plastic component $d u_{t, j}^{p}(t)$ so that:

$$
d u_{t, j}^{p}(t)=d u_{t, j}(t)-d u_{t, j}^{e}(t)
$$

with

$$
d u_{t, j}^{e}(t)=-\frac{d F_{t, j}(t)}{K_{t}}
$$

the energy $E_{d}(D)$ dissipated within the domain $D$ can be defined from an incremental expression as follows:

$$
d E_{d}(D)=-\sum_{j=1}^{N_{c}(t)}\left(F_{t, j}(t) d u_{t, j}^{p}(t)\right)
$$

Likewise, the strain energy $E_{s}(D)$ associated with all contacts $N_{c}(t)$ located inside the domain $D$ at time $t$, under an incremental formalism, reads:

$$
d E_{s}(D)=\sum_{j=1}^{N_{c}(t)}\left(F_{n, j}(t) d u_{n, j}(t)-F_{t, j}(t) d u_{t, j}^{e}(t)\right.
$$

where $F_{n, j}(t)$ and $F_{t, j}(t)$ are the normal and tangential forces associated with the $\mathrm{j}^{\text {th }}$ contact of the domain at time $t$; $d u_{n, j}(t)$ is the normal displacement increment corresponding to the contact ' $j$ ' at time $t$.

The relative magnitude of energies depends to a large extent on the local spatial configuration of the particles. In order to limit the local effects related to the local particles' layout, simulations are carried out for various local configurations, which can be done from the same granular assembly describing the soil 
by considering $N$ different impact points. A minimum distance $d_{i}$ between two impact points is set to limit an unnecessary refinement of the results. Numerical investigations show that simulations using $N=100$ impact points $d_{i}=0.05 \mathrm{~m}$ apart are necessary to obtain stable mean values and standard deviations of the kinematic parameters reflected (Figure 10). These conditions are used to ensure the reproducibility of the results and accurately evaluate local variability (Figure 10).

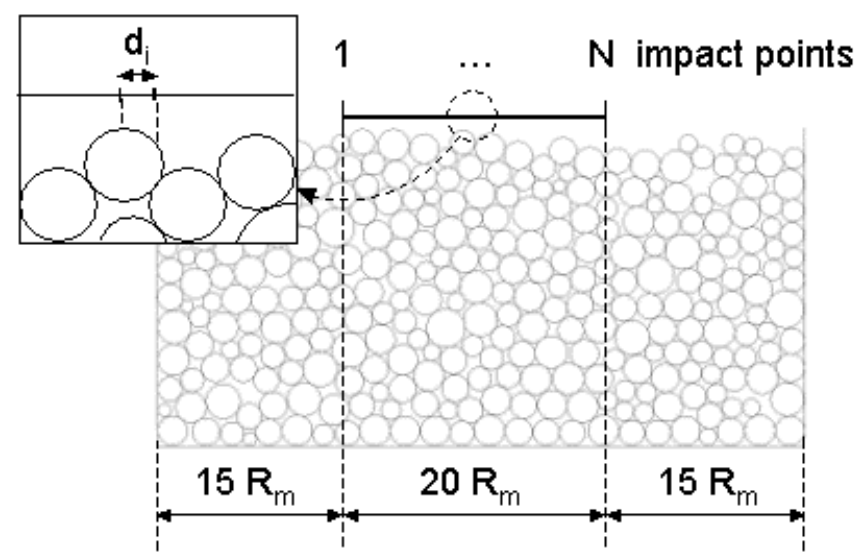

Figure 9. Location of impact points. When considering the impact points' range, different local conditions (particle arrangements) are encountered.

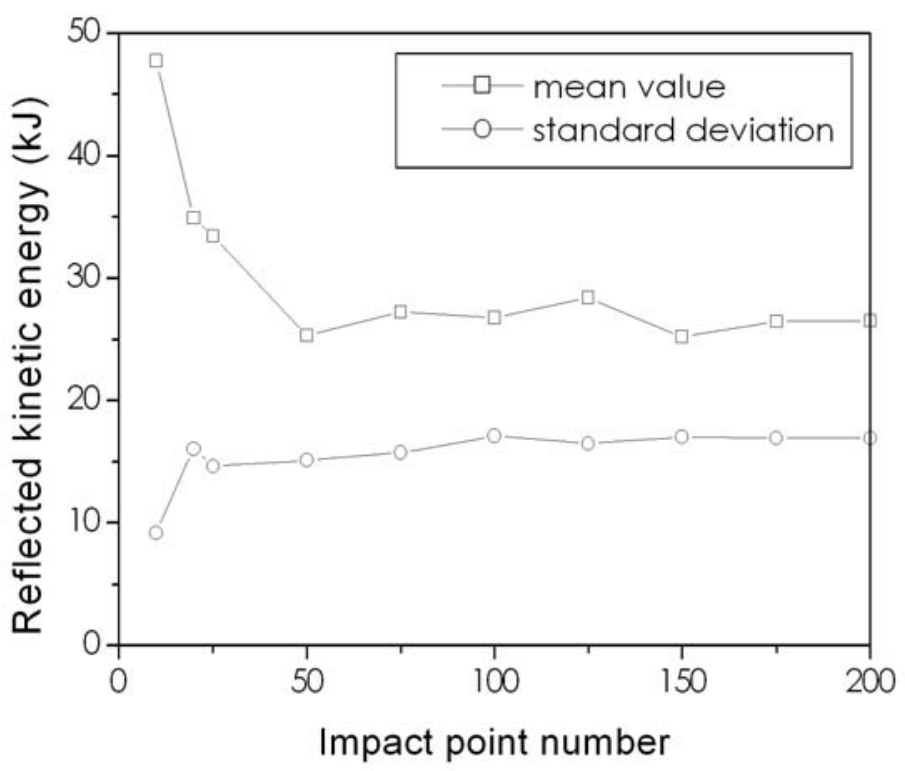

Figure 10. Minimum impact point number to ensure reproducibility of results. 


\subsection{Validation of the numerical simulation procedure}

In order to evaluate the relevance of the numerical model, experimental results from the literature were compared to the results of simulations under similar conditions.

Experimental results obtained further to a detailed 2D and 3D experimental study of the collision of an incident bead on a granular packing (Rioual et al., 2000; Rioual et al., 2003; Oger et al., 2005; Beladjine et al., 2007; Crassous et al., 2007) are compared first. In the experimental study, the granular packing is composed of beads that are the same size as the impacting particle. However, important differences exist between these experiments and the simulation procedure defined in this paper. The size of the particles, the polydispersity of the granular material, the incident velocity range, and the particles' mechanical and geometrical characteristics are not the same.

The experimental results obtained in Rioual et al. (2000) and Beladjine et al. (2007) describe the impact of a PVC bead on a slightly polydisperse granular material also composed of PVC beads. The range of the incident velocity $V_{i}$ is defined compared to the radius of the impacting particle by the intermediate of the Froude number $F r$ :

$F r=\frac{V_{i}}{\sqrt{2 g R_{b}}}$

The incident velocity range used in the experiments is $50<F r<250$, which is very different from the range used in our simulations $(2<F r<15)$.

During the experimental campaign conducted by Beladjine et al. (2007), 100 experiments were conducted for each set of incident parameters in order to provide results relevant for the variability of the impact phenomenon. For the numerical simulations, we therefore also choose to perform impacts on 100 different impact points defined according to the methodology developed in the previous section for each set of parameters.

Although the differences between the experimental setup and our numerical model are important, the results obtained in both cases can be compared. In particular, the normal restitution coefficient $e_{n}$, the restitution coefficient $e$ and the reflected angle $\alpha_{r}$ can be compared for varying incidence angles $\alpha_{i}$ :

$e_{n}=\frac{V_{n r}}{V_{n i}}$ 
$e=\frac{V_{r}}{V_{i}}$

$\alpha_{r}=\arccos \left(\frac{V_{n r}}{V_{r}}\right)$

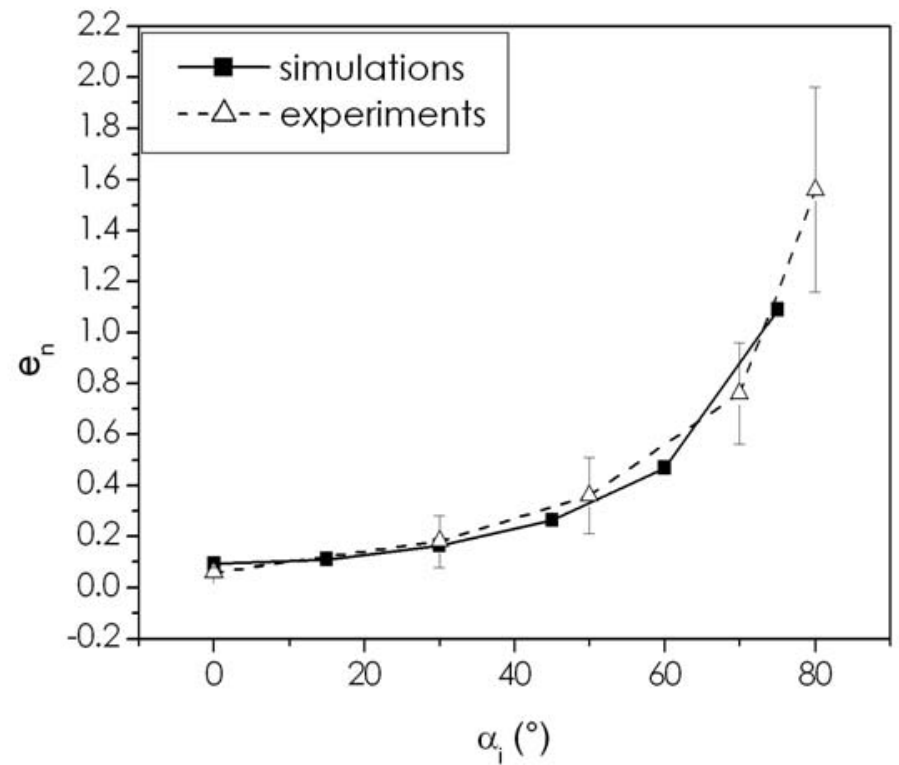

Figure 11. Comparison between simulations and experiments (after Beladjine et al. 2007) for the mean value of coefficient $e_{n}$.

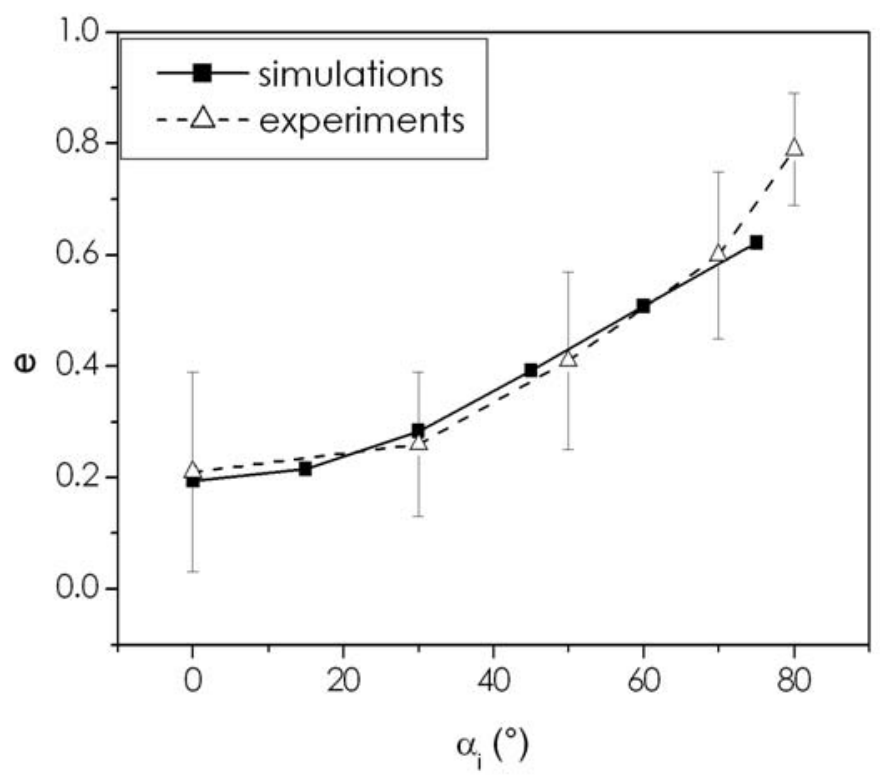


Figure 12. Comparison between simulations and experiments (after Beladjine et al. 2007) for the mean value of coefficient $e$.

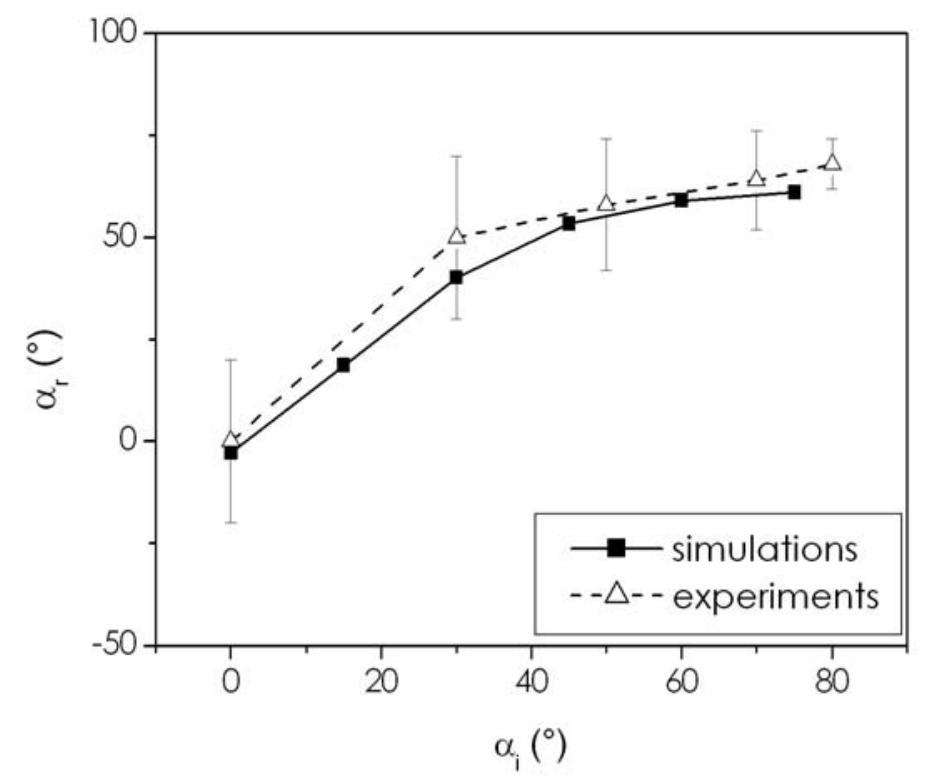

Figure 13. Comparison between simulations and experiments (after Beladjine et al. 2007) for the mean value of the reflected angle $\alpha_{r}$.

The comparison between the experiments and our simulations clearly shows that the mean values obtained are nearly the same for all the parameters compared. The numerical simulation procedure developed is therefore relevant for modelling the impact of a particle on a granular material when the size of the impacting particle is approximately the same as the size of the soil particles.

Since, in the study planned, the boulder's radius can vary up to $10 R_{m}$, the numerical results from our model are also compared to the results of an experimental campaign consisting of the penetration of a sphere inside a granular packing with $R_{b} \approx 9 R_{m}$ (Ciamarra et al., 2004). In these experiments, the particle impacted the sample perpendicular to the soil surface. Moreover, the incident velocity of the impacting particle varied from $1.12 \mathrm{~m} / \mathrm{s}$ to $3.63 \mathrm{~m} / \mathrm{s}$ and the radius of the impacting particles was $R_{b}=2.23 \mathrm{~cm}$. As a consequence, the Froude number ranged from 1.7 to 5.5 . The soil sample was composed of spherical particles with a mean radius $R_{m}=0.242 \mathrm{~cm}$. Moreover, the granular packing comprised two particle types that had two slightly different radii to reduce crystallization. The width of the sample was approximately $420 R_{m}$ and its height was approximately $120 R_{m}$. 
The aim of the comparison is to evaluate whether the numerical procedure defined in section 2.2 provides the same results for the changes in the projectile's penetration and normal velocity versus time as observed in Ciamarra et al. 2004. For these comparisons, impact is simulated using our numerical model under similar conditions. First, the sizes of the particles (minimum, maximum and mean), the size of the sample and the incident velocity of the impacting particle are set at the same values as in Ciamarra et al. 2004. However, the packing is a polydisperse assembly of spheres instead of a bidisperse assembly. In addition, the mechanical properties of the soil particles defined in section 2.2 are conserved.

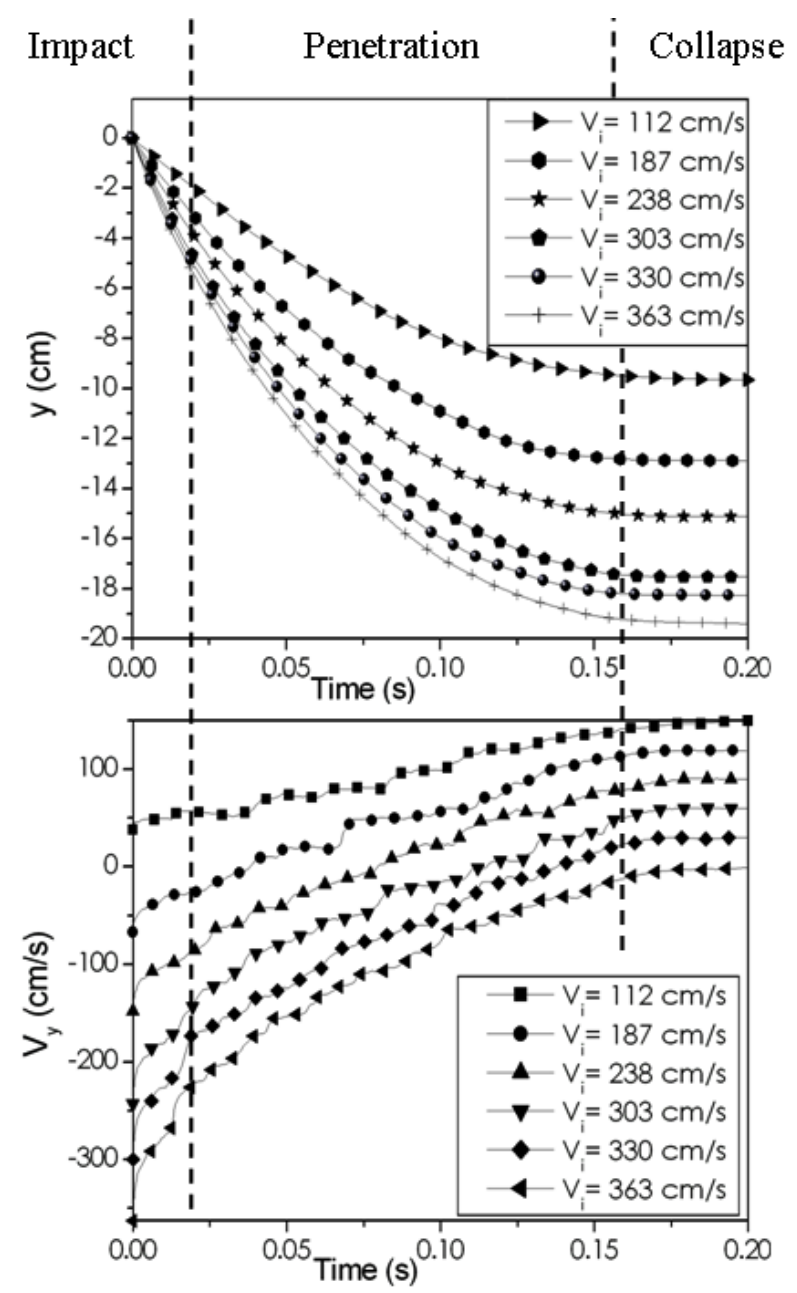

Figure 14. Simulation results for the penetration $y(t)$ and the normal velocity $V_{y}(t)$ of the projectile versus time (on the graph $V_{y}(t)$ ) the ordinate for each successive impact velocity $V_{i}<363 \mathrm{~cm} / \mathrm{s}$ is shifted by $30 \mathrm{~cm} / \mathrm{s}$ for clarity). 

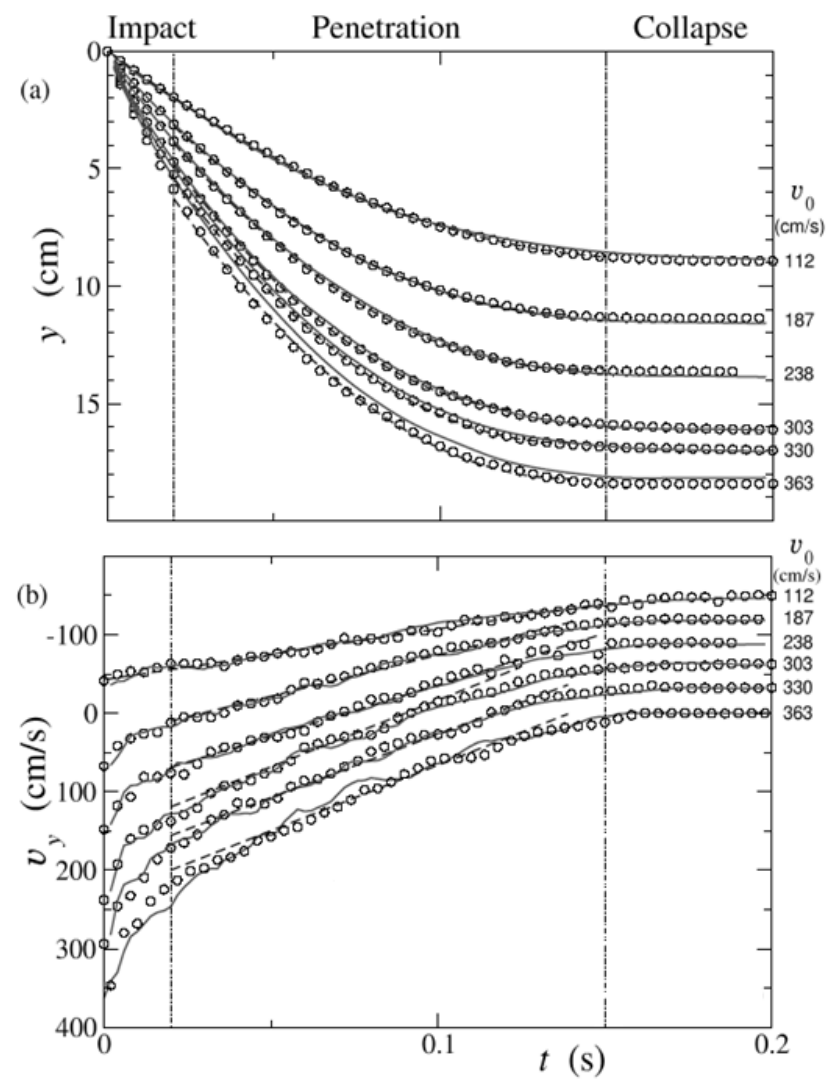

Figure 15. After Ciamarra et al. 2004: Position $y(t)$ (a) and velocity $v_{y}(t)$ (b) of the projectile as a function of time for different impact velocities, from experiment ( ${ }^{\circ}$ (solid lines are the results of simulations conducted by Ciamarra et al. 2004). The two vertical dot-dashed lines give approximate boundaries between three regions: impact, penetration, and collapse. The ordinate for (b) for each successive impact velocity $v_{0}<363 \mathrm{~cm} / \mathrm{s}$ is shifted by $30 \mathrm{~cm} / \mathrm{s}$ for clarity.

The comparison to the results obtained in Ciamarra et al. (2004) (Figure 15) shows that both the penetration and the normal velocity of the projectile (Figure 14) are qualitatively the same in the experiments and the numerical simulation. In particular, the three phases of motion are observed: the impact of the projectile, the penetration of the projectile and the collapse of the transient crater after the projectile has stopped (Figure 14). Moreover, the large deceleration of the projectile during the impact phase, the description of $y(t)$ as a parabola during the penetration phase and the fact that the time taken for the projectile to decelerate to a stop was independent of the incident velocity were observed in both cases. Finally, the approximately linear decrease in the projectile's velocity during the penetration phase was also observed both in the simulations and in the experiments (Figure 14). However, slight quantitative differences were observed for the maximum penetration of the impacting particle and for the 
time taken to reach the collapse phase. This difference might be caused by differences in the grading curves (polydisperse instead of bidisperse packing), the different formulations of the contact law or differences in the stiffness of particles.

The comparison between the experiments (Beladjine et al. 2007; Ciamarra et al. 2004) and the numerical simulations conducted under similar conditions shows the relevance of the model defined in section 2.2 for the impact on granular materials composed of spherical particles for which the mechanical properties are well known, even though the relevance of the numerical model for real rockfall events has not yet been established conclusively. The correct predictions of the numerical simulations in the previous case means that the numerical model can be assumed to be relevant for the first-order phenomena leading the impact of a boulder on a scree slope when the boulder size is approximately the same as the soil particle size and when contact attrition and rock breakage are negligible.

\section{Energy propagation process}

General energy propagation processes are deduced from several impact simulations for approximately identical values of $R_{m}$ and $R_{b}$. For each impact simulation, a predefined soil type is chosen and is impacted by a spherical impacting particle. The varying parameters are sample size, impacting particle radius and the initial kinematic parameters. The magnitude of all energies involved during impact strongly depends on the local spatial configuration of the particles. For each set of incident kinematic parameters, several impact simulations were therefore held for 100 different impact points in accordance with the methodology defined in section 2.3 and the analysis of the result was based on the study of the mean values of all quantities.

\subsection{Energy balance}

The impact of the particle on the soil is composed, in general, of successive contact interactions between the impacting particle and soil particles. Each contact interaction is characterized by an impacting particle’s kinetic energy variation (Figure 16). The first contact interaction between the impacting particle and the soil particles constitutes the first phase of the impact process, which is very important because most of the impacting particle's incident energy is transferred to the soil during this contact interaction (Figure 16). 


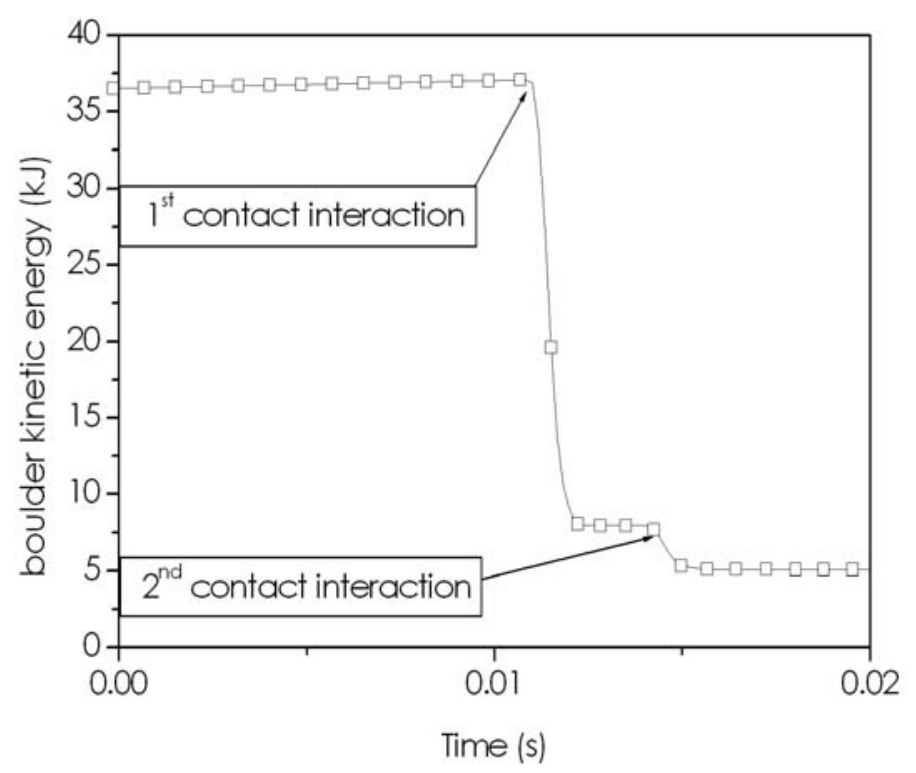

Figure 16. Impacting particle's kinetic energy as it evolves during the impact phase.

The first phase of the impact process highlights a partial energy exchange from the block to the soil sample. Although the impacting particle's incident energy $E_{k i}$ is substantially reduced during this phase, it must be noted that the block's kinetic energy is not null at the end of the first phase. The incident impacting particle energy $E_{k i}$ is transferred over this phase into different energy types. Four types of energy are involved over this first stage: the impacting particle’s kinetic energy, the soil particle’s kinetic energy, the soil’s elastic strain energy and the dissipated energy.

It can be shown from discrete element simulations that the energy dissipated through frictional processes during the first phase is less than $1 \%$ of the incident energy. This result is valid whatever the incident kinematic conditions.

Moreover, the initial energy transfer is only slightly influenced by the incident velocity over the velocity range explored (Figure 17). At the end of the first phase, the ratio of the mean of the impacting particle's reduction in kinetic energy $\left(E_{k i}-E_{k f}\right)$ to the incident kinetic energy is nearly the same whatever the incident velocity $V_{i}$ (Figure 17) because the local contact law formulation is rate-independent and the energy dissipated through frictional processes during the first phase is low. 


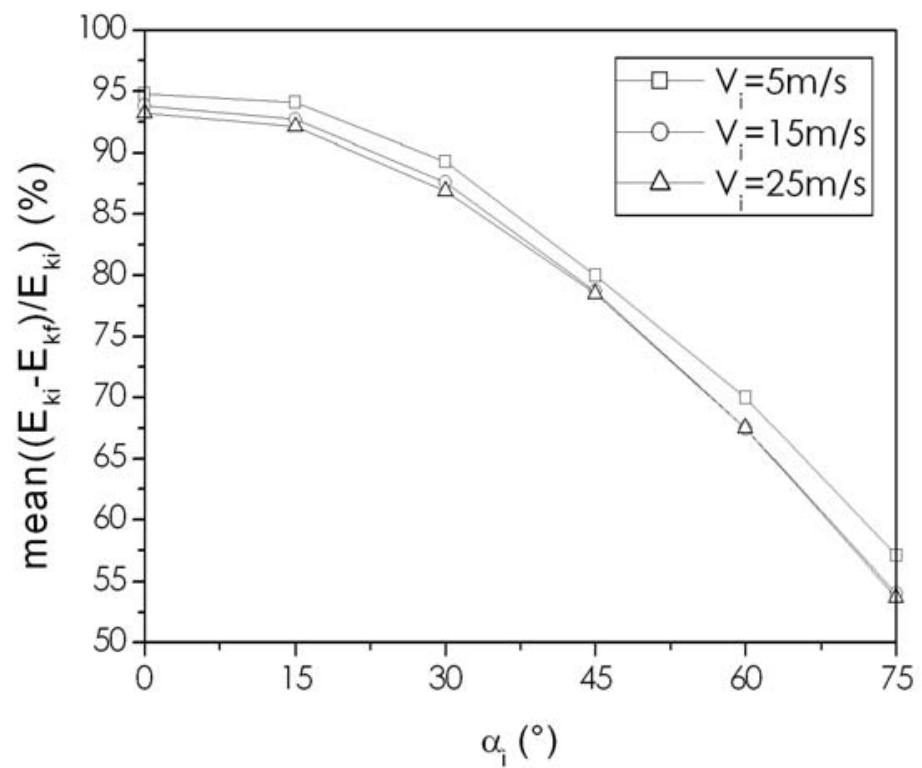

Figure 17. Impacting particle's mean kinetic energy reduction for varying incident angles and velocities

$$
\left(R_{b}=1.33 R_{m}\right) \text {. }
$$

On the other hand, the impacting particle's kinetic energy at the end of the first phase depends to a large extent on the incidence angle $\alpha_{i}$. The impacting particle's kinetic energy reduction is greater for normal ( $\alpha_{i}=0^{\circ}$ ) impacts than for grazing $\left(\alpha_{i}=75^{\circ}\right)$ impacts (Figure 17). A microscopic investigation (on the particle scale) is relevant to interpret this result.

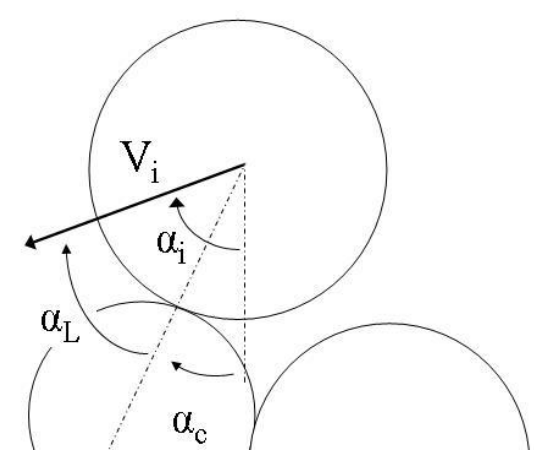

Figure 18. Local impact angle definition.

Geometrical investigations show that for varying impact points both the local impact angle $\alpha_{c}$ and the local incidence angle $\alpha_{L}$ (Figure 18) vary. When the incidence angle $\alpha_{i}$ increases, the local incidence 
angle $\alpha_{L}$ probability density function is translated towards larger values of $\alpha_{L}$ (Figure 19). In other words, the incidence angle increase induces an increase in the mean local incidence angle. Since the relative magnitude of normal and tangential contact forces is related to the local incidence angle, the previous property induces the force in the normal direction to contact to reduce for increasing incidence angles. In addition, since the energy transfer results mainly from force transfers in the normal direction to contact, the transferred energy decreases for increasing incidence angles. As a result, the mean impacting particle’s kinetic energy reduction is lower for a large incidence angle, as shown in Figure 17.

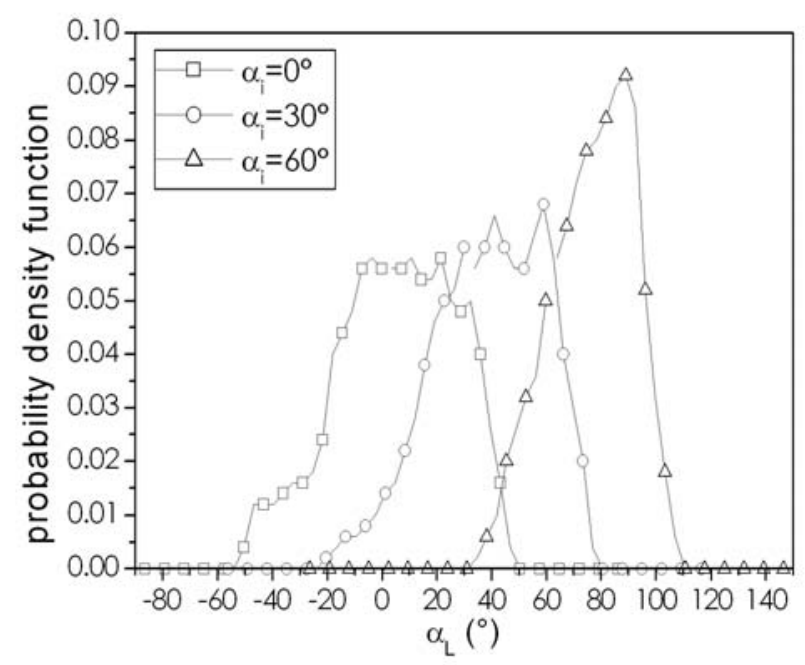

Figure 19. Local incidence angle as it evolves for varying incidence angles $\left(R_{m}=R_{b}\right)$.

The simulations also show that impacting particle behaviour depends a great deal on the $R_{b} / R_{m}$ size ratio. This parameter not only acts on energy transfer, but also influences the direction of the impacting particle after the first phase.

If the impacting particle's radius is smaller than the mean particle radius $\left(R_{b} / R_{m}<1\right)$, the impacting particle's normal velocity directly inverses; the mean of the ratio of the final impacting particle's normal velocity $V_{n f}$ to the incident impacting particle's normal velocity $V_{n i}$ is negative (Figure 20). For intermediate values $\left(1<R_{b} / R_{m}<3.5\right)$, the same behaviour is observed for grazing impacts only $\left(\alpha_{i}=75^{\circ}\right)$. On the contrary, for small incidence angles, the mean of the $V_{n f} / V_{n i}$ ratio becomes positive, meaning that the impacting particle’s normal velocity $V_{n}$ does not inverse (Figure 20). For higher values 
of the size ratio ( $R_{b} / R_{m}>3.5$ ), the impacting particle's normal velocity sign no longer changes for any incidence angle. It can be noted that the dependence on the incidence angle previously highlighted (Figure 17) exists for any impacting particle size (Figure 21), but it decreases as $R_{b} / R_{m}$ increases.

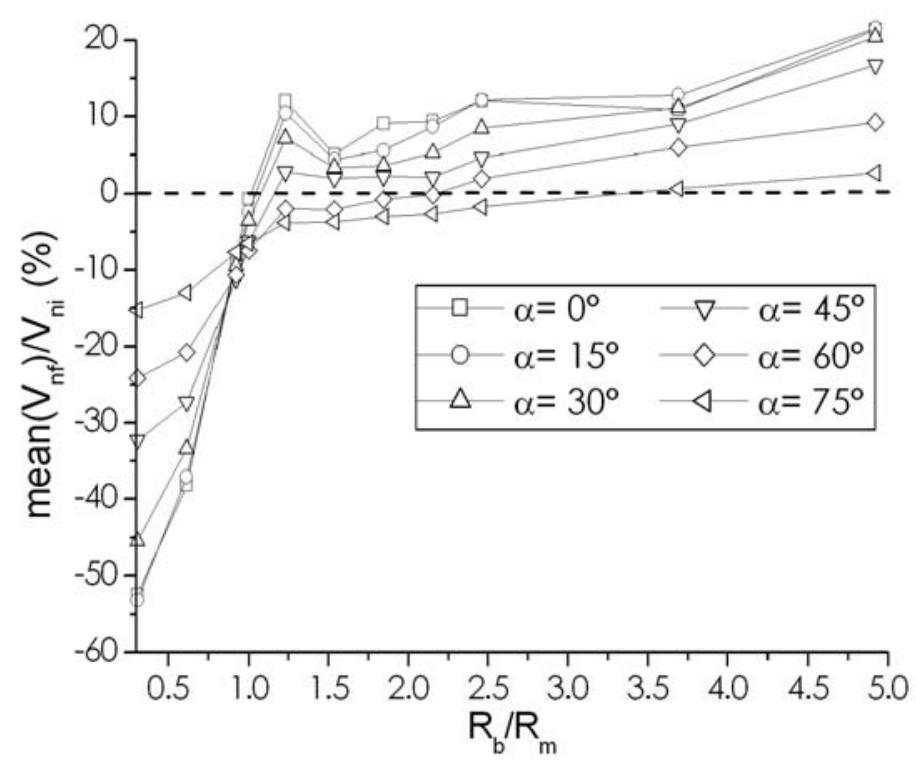

Figure 20. Impacting particle's mean final normal velocity for varying $R_{b} / R_{m}$ ratio and for fixed $E_{k i}$ value.

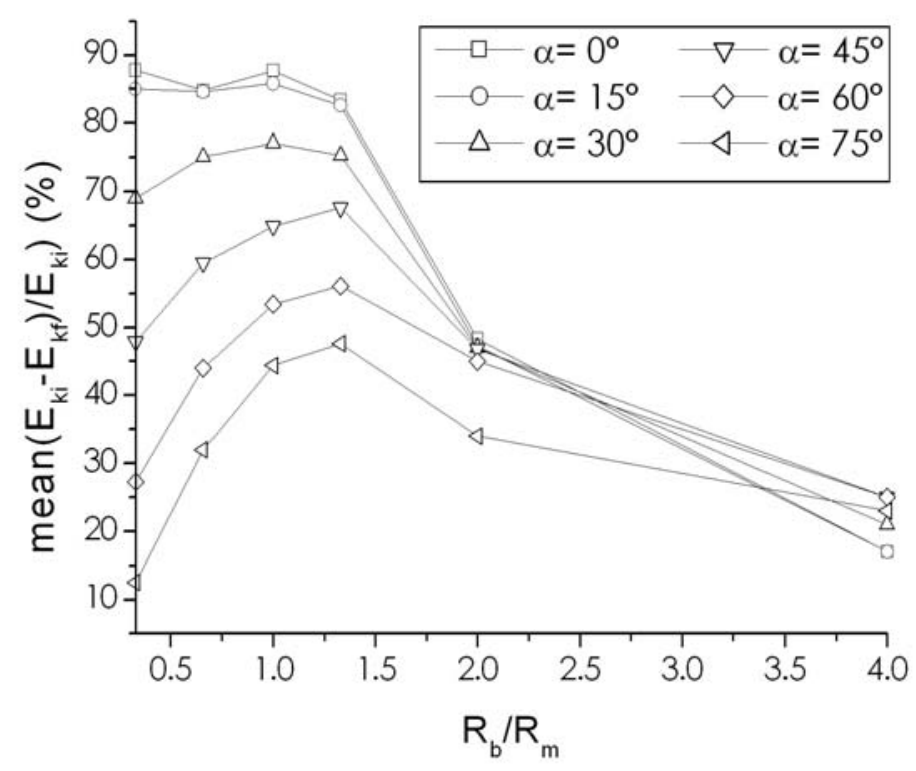


Figure 21. Impacting particle's kinetic energy reduction for varying $R_{b} / R_{m}$ ratio.

These results can be qualitatively approached assuming that the initial impact process can be described by the binary impact of one particle on another that was initially idle since the impact loading induces contact forces much larger than the mean of the contact forces before impact. The physics of binary impacts is very complex, involving both the kinematics and mechanical properties of particles (Goldsmith 1960; Frémond 1995; Thornton and Ning 1998; Stronge 2000).

Herein, the elastic normal impact of a rigid particle on another rigid particle which is initially idle is considered. A relation linking the incident and the reflected velocities of the impacting particle can be established. Since the impact is elastic, the kinetic energy is conserved during the impact process:

$\frac{1}{2} m_{b} V_{b i}^{2}=\frac{1}{2} m_{b} V_{b f}^{2}+\frac{1}{2} m_{p} V_{p f}^{2}$

where $V_{b i}$ and $V_{b f}$ are the velocities of the impacting particle at the beginning and the end of the first phase; $m_{b}$ is the impacting particle's mass; $m_{p}$ is the impacted particle's mass and $V_{p f}$ is the impacted particle's velocity at the end of the first phase.

The percussion theory (see for instance Frémond 1995) relates the linear momentum variation of the impacting particle to the impulse of the particle on the impacting particle $\Pi_{p / b}$ :

$m_{b}\left(V_{b i}-V_{b f}\right)=\Pi_{p / b}$

The same relation can be written between the linear momentum variation of the particle to the impulse of the impacting particle on the particle $\Pi_{b / p}$ :

$-m_{p} V_{p f}=\Pi_{b / p}$

Since $\Pi_{b / p}=-\Pi_{p / b}$, the combination of Eqs. (20) and (21) expresses $V_{p f}$ as a function of $V_{b i}$ and $V_{b f}$ :

$V_{p f}=\frac{m_{b}}{m_{p}}\left(V_{b f}-V_{b i}\right)$

Moreover, Eq. (19) can be rewritten in order to express $V_{p f}$ versus $V_{b i}$ and $V_{b f}$ :

$V_{p f}^{2}=-\frac{m_{b}}{m_{p}}\left(V_{b f}^{2}-V_{b i}^{2}\right)$ 
The combination of Eqs. (22) and (23) leads to the relation relating the incident and the reflected velocities of the impacting particle:

$$
\frac{V_{b f}}{V_{b i}}=\frac{\frac{m_{b}}{m_{p}}-1}{1+\frac{m_{b}}{m_{p}}}
$$

If we consider that all particles have the same density, the previous relation can be expressed using $R_{b}$ and $R_{m}$ :

$$
\frac{V_{b f}}{V_{b i}}=\frac{\frac{R_{b}^{3}}{R_{m}^{3}}-1}{1+\frac{R_{b}^{3}}{R_{m}^{3}}}
$$

This relation shows that, for small impacting particles $\left(R_{b} / R_{m}<1\right)$, the impacting particle's normal velocity sign changes during the first phase. On the contrary, if $R_{b}$ is higher than $R_{m}$, the impacting particle's normal velocity retains the same direction.

The percentage of the impacting particle's kinetic energy reduction $\Delta E_{k}$ can be expressed as follows:

$$
\Delta E_{k}=\frac{E_{k i}-E_{k f}}{E_{k i}}
$$

where $E_{k f}$ is the impacting particle's final kinetic energy and $E_{k i}$ is the impacting particle's incident kinetic energy.

Assuming that all particles have the same density, the impacting particle's kinetic energy reduction $\Delta E_{k}$ can be related to the final and incident impacting particle velocity:

$$
\Delta E_{k}=1-\left(\frac{V_{b f}}{V_{b i}}\right)^{2}
$$

Given Eq. (25), the expression of the impacting particle’s kinetic energy reduction $\Delta E_{k}$ is: 


$$
\Delta E_{k}=\frac{4 \frac{R_{b}^{3}}{R_{m}^{3}}}{\left(1+\frac{R_{b}^{3}}{R_{m}^{3}}\right)^{2}}
$$

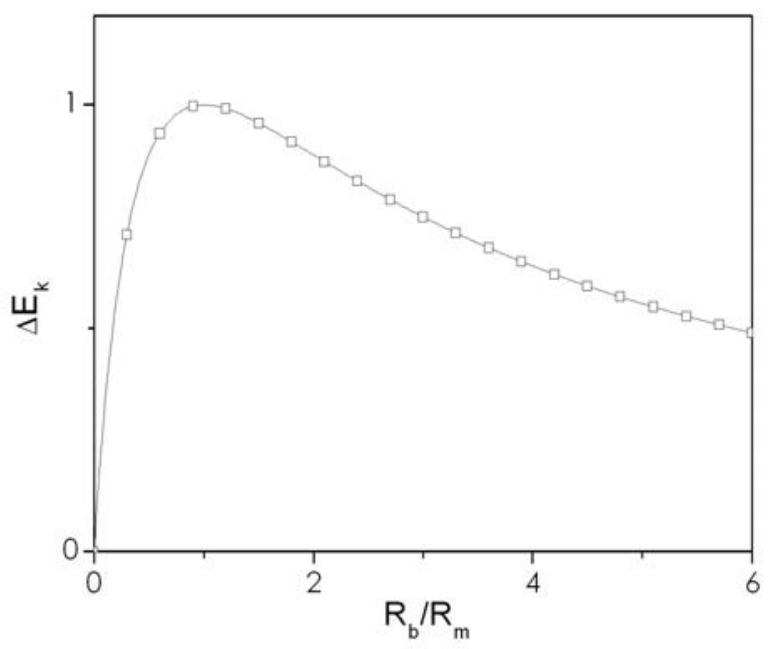

Figure 22. Impacting particle's kinetic energy reduction versus $R_{b} / R_{m}$.

Equation (28) emphasizes that, while the impacting particle's size is smaller than the mean size of the soil particles, the reduction in the impacting particle's kinetic energy increases as the impacted particle's size increases (Figure 22). Moreover, the dependence of particle size on conserved energy inverses if $R_{b}$ is higher than $R_{m}$. These results (Figure 22) are in accordance with the simulation results (Figure 21).

This simple analogy does not account for the influence of the incidence angle. However, as determined previously (Figure 17), the impacting particle's kinetic energy reduction is larger for normal impacts than for grazing impacts (Figure 21).

\subsection{Shock wave propagation}

The beginning of the impact process gives rise to a sudden increase in the total energy of the soil due to the first energy transfer from the impacting particle to the soil. The energy transferred to the soil is dissipated as soon as it is introduced in the sample (Figure 23). Dissipation is related to frictional processes that occur between adjoining particles by sliding, rolling and contact opening. 


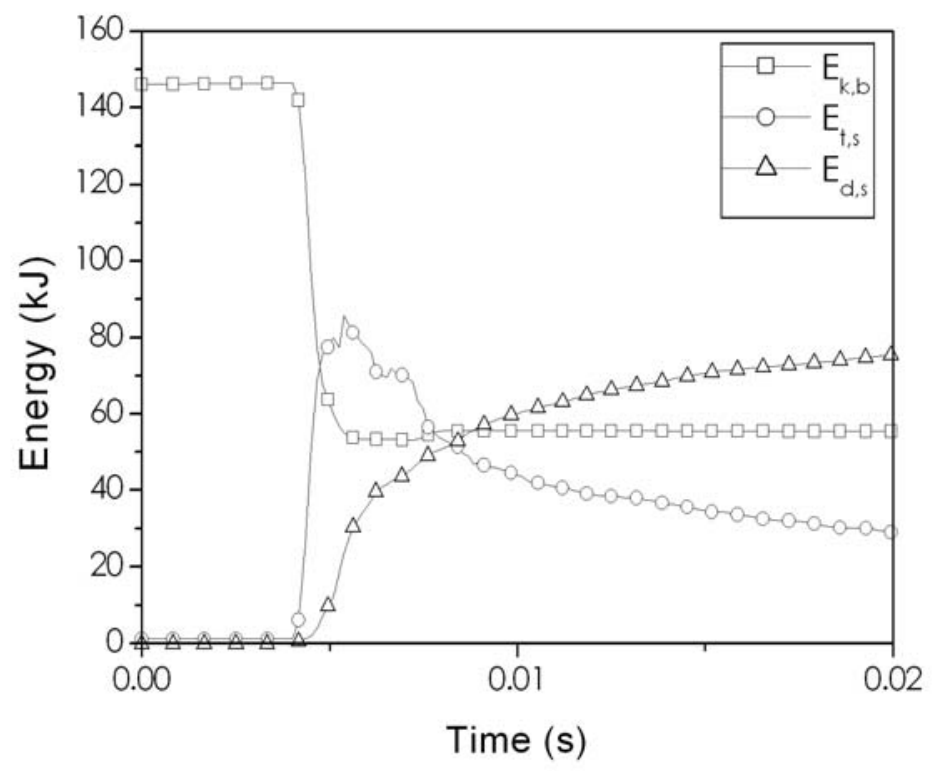

Figure 23. Impacting particle's energy $\left(E_{k, b}\right)$, the total sample's energy $\left(E_{t, s}\right)$ and the energy dissipated $\left(E_{d, s}\right)$ over time.

In order to characterize energy propagation, the evolution of all energies during the impact phase is observed on concentric crowns centred on the impact point (Figure 24).

The sample depth is set at $20 R_{b}$ from the impact point so that energy transfers can be observed over a wide domain and the boundary's influence can be prevented. The impact points are chosen at least $20 R_{b}$ from the sample's lateral boundaries for the same reason. Simulations are carried out for several impact points to account for the variability in the local soil configuration. The following phenomena are observed for all impact conditions.

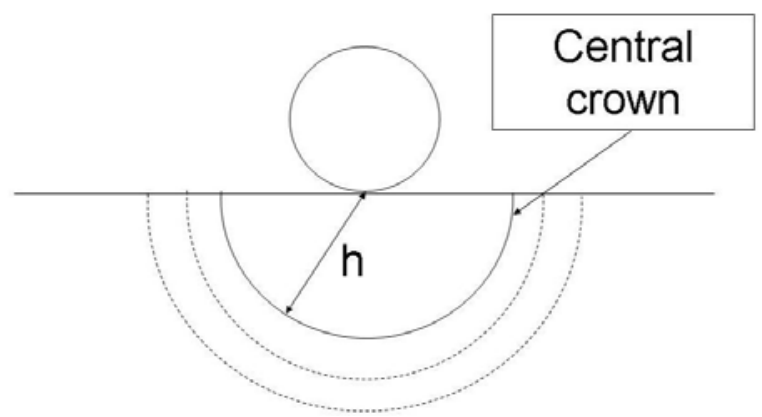

Figure 24. Concentric crowns centred on the impact point. All crowns are characterized by the radius $h$. 
The strain energy peak observed on the central crown just after the first energy transfer (Figure 25) shows that the energy transferred to the soil at the beginning of the impact is first converted into strain energy near the impact point.

The strain energy peak is directly followed by a kinetic energy peak near the impact point (Figure 25). The initial stage can therefore be divided into two successive phases:

- a compression phase associated with a local strain energy peak on the central crown,

- a restitution phase inducing a kinetic energy peak near the impact point.

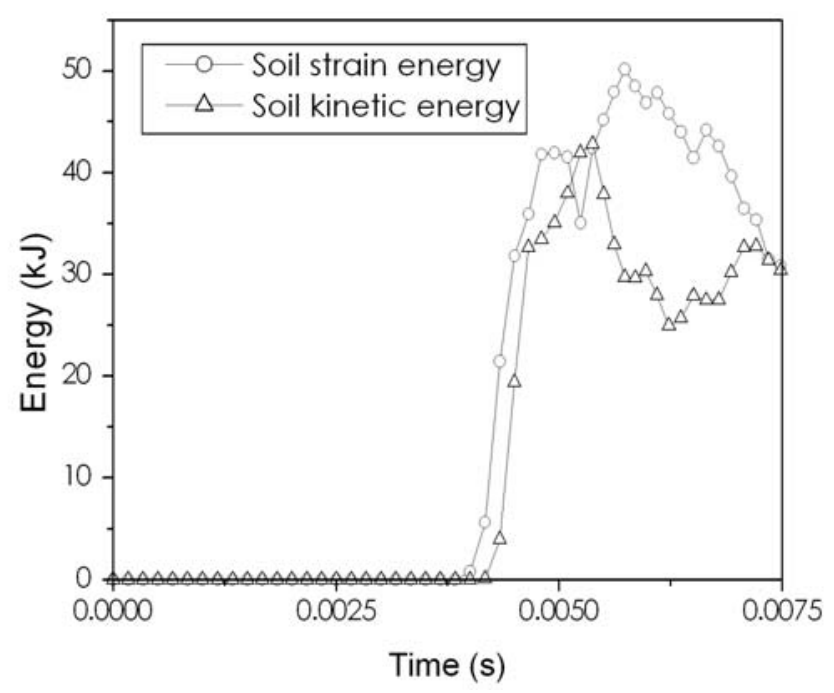

Figure 25. Strain and kinetic energy peaks.
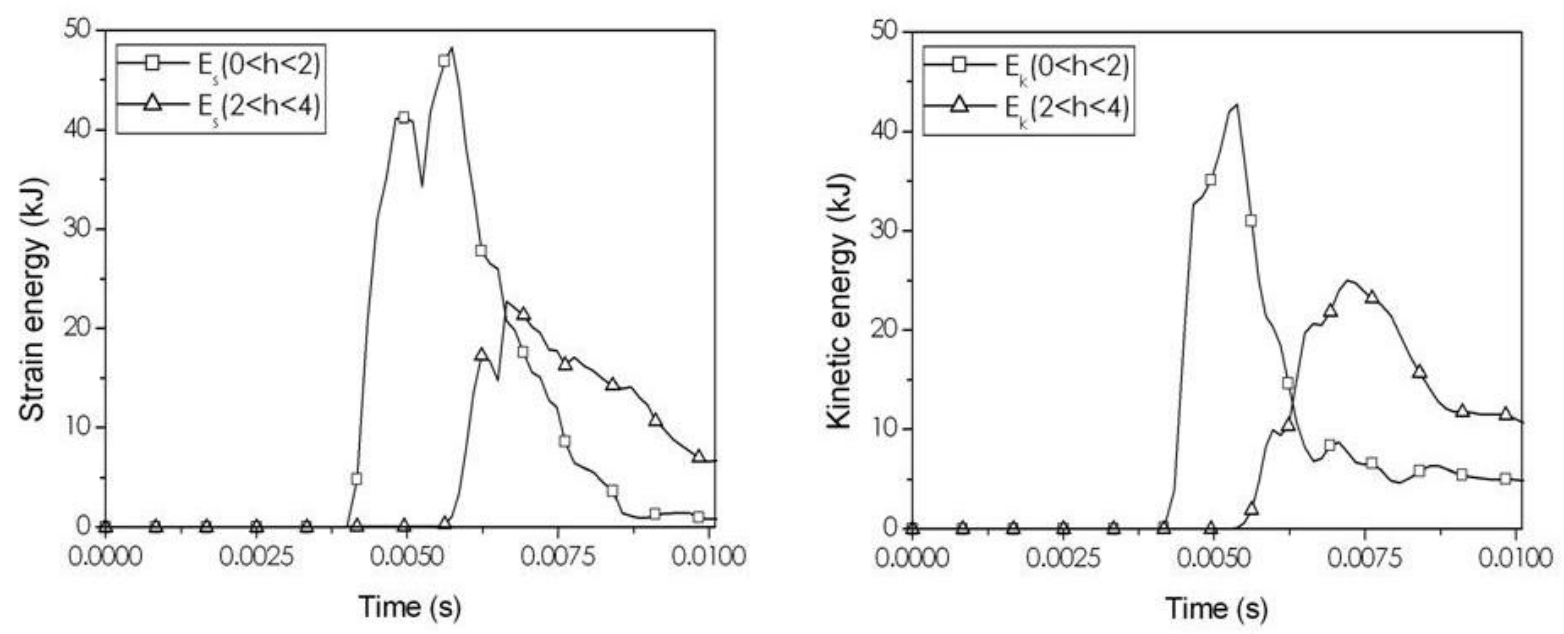

Figure 26. The time difference of the strain and kinetic energy peaks on concentric crowns. 


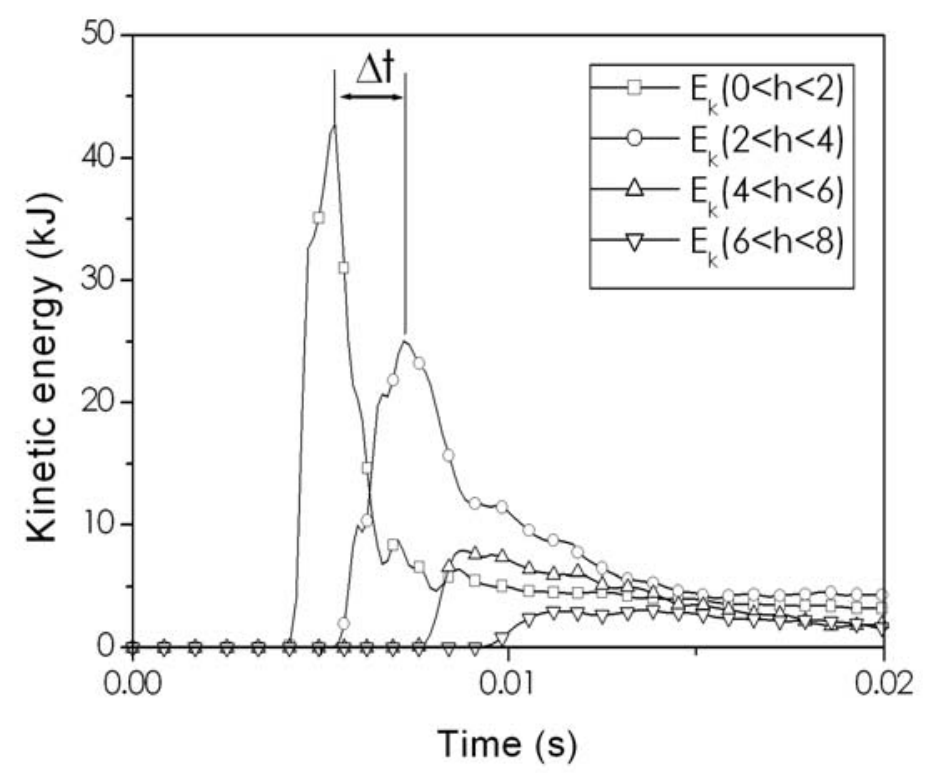

Figure 27. Kinetic energy over time on concentric crowns.

The kinetic energy peak for the particles near the impact point shows that the initial energy transfer brings the particles of the central crown out of equilibrium, inducing their movement. The displacement of the previous particles also brings the particles of the second crown out of equilibrium. This leads to a strain energy peak followed by a kinetic energy peak (Figure 26) on the second concentric crown. The energy peaks are time-delayed from one crown to the adjacent one (Figure 27). This phenomenon has already been described based on theoretical, numerical and experimental results on a one-dimensional column of beads (Campbell 2003; Coste et al. 1997; Job et al. 2007) and two-dimensional media (Somfai et al. 2006; Hostler and Brennen 2005; Sadd et al. 2000). This is relevant for shockwave propagation. The shockwave velocity can be calculated using the time difference between peak occurrences on two adjacent crowns. The shockwave covers the distance $d_{c}$ between the inner diameters of two adjacent crowns during a time lapse $\Delta t$ corresponding to the time difference between the kinetic energy peak occurrence on these consecutive crowns (Figure 27). The wave speed magnitude $c$ is therefore the ratio of the distance $d_{c}$ to the time difference $\Delta t: c=d_{c} / \Delta t$. Whatever the local configuration of the soil particles and the incident kinematic conditions, the magnitude of the wave speed is always on the order of $1000 \mathrm{~m} / \mathrm{s}$. 


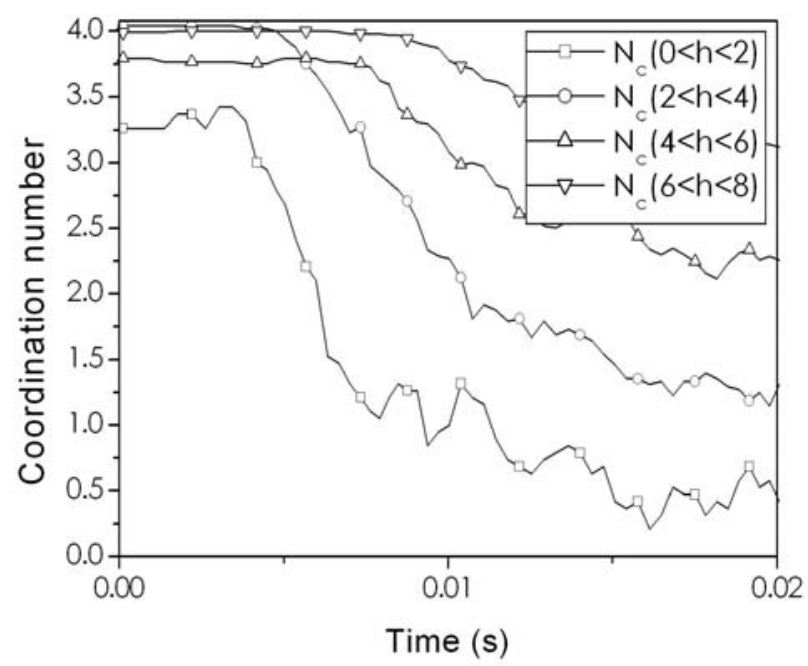

Figure 28. Coordination number on concentric crowns evolving over time.

Shockwave propagation simultaneously occurs with a substantial decrease in the coordination number $\mathrm{N}_{\mathrm{c}}$ on the crown crossed by the wave (Figure 28). A similar phenomenon has already been described for a one-dimensional column of beads (Campbell 2003) and two-dimensional media (Hostler and Brennen 2005). A decrease in the coordination number occurs in our context because the disturbing force is much larger than the mean force between particles inside the initial sample (Campbell 2003). In this case, the high magnitude of the energy introduced induces a total change in the initial contact force network when the shockwave crosses. Before the shockwave arrives, the soil is in equilibrium and is composed of two contact phases: the strongly loaded contact phase and the weakly loaded one (Radjai et al. 1998). A contact is part of the strongly loaded phase when the associated contact force is higher than the mean contact force in the sample. Otherwise, it is part of the weakly loaded phase. At the beginning of the impact process, given that the disturbing force is much larger than the mean contact force before impact, the strongly loaded contact phase is concentrated near the impact point at impact time $\mathrm{T}_{0}$. During the compression wave propagation, contact forces close to the wave front are very high compared to other contact forces. The strongly loaded phase is then concentrated near the wave front and the other contacts of the sample are part of the weakly loaded phase (Figure 29). In addition, contact openings occur just after the wave front has passed (Campbell 2003), leading to a substantial decrease in the coordination number. 


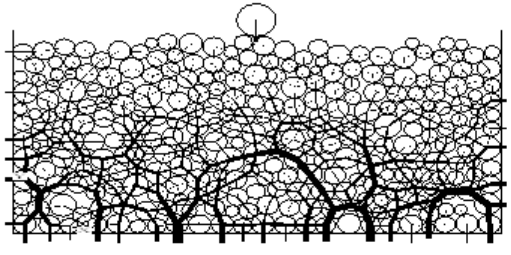

$\mathrm{t}=0 \mathrm{~s}$

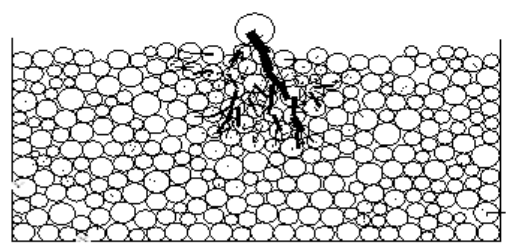

$\mathrm{t}=0.0037 \mathrm{~s}$

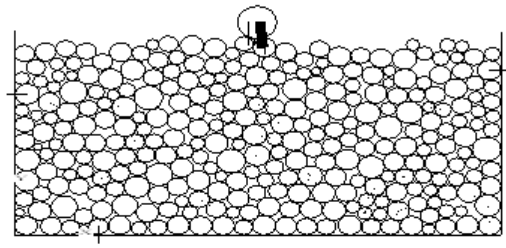

$\mathrm{t}=0.0012 \mathrm{~s}$

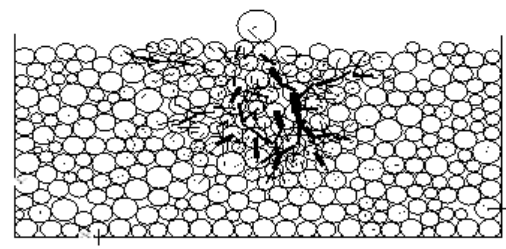

$\mathrm{t}=0.005 \mathrm{~s}$

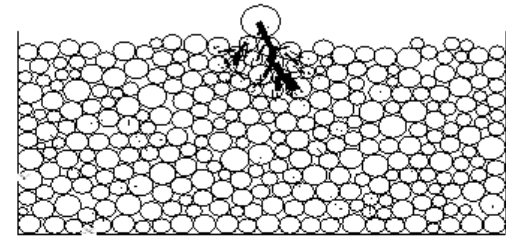

$\mathrm{t}=0.0025 \mathrm{~s}$

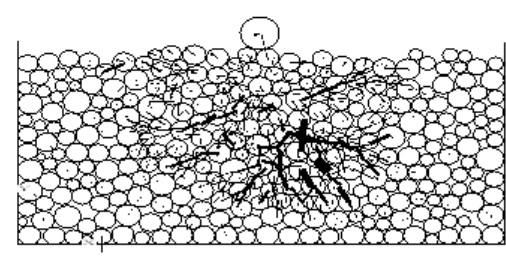

$\mathrm{t}=0.0062 \mathrm{~s}$

Figure 29. Snapshots of compression wave propagation.

The decrease in the coordination number and the localization of the strongly loaded phase due to shockwave crossing are relevant to a local fluidization of the soil due to shockwave propagation. After shockwave propagation, as the fluidization process persists over a period longer than the impact duration, the interaction between the impacting particle and the soil near the sample surface is therefore equivalent to one particle with a granular fluid.

It can be noted that the local fluidization of the soil is more pronounced in the simulations presented in this paper than in real occurrences because in the numerical model, energy is only dissipated through frictional processes, whereas, in real cases, energy is also dissipated during the interaction of two rocks by local yielding near the contact surface, crack propagation or rock breakage.

\subsection{Influence of boundary conditions}

In practical cases, such as when the impacted soil is not infinite, the impacting particle's behaviour is influenced by the bedrock under the coarse soil. The DEM model considered throughout the previous section can be used to investigate the influence of rigid bedrocks on the impact process. The bedrock is then modelled by the bottom wall of the sample. The displacement of a shockwave from the soil surface to the sample bottom can easily be characterized. However, after the shockwave has reached the bottom of the sample, the energy propagation cannot be clearly identified since it propagates through a fluidized medium composed of moving particles. Nevertheless, the amount of energy reflected on the sample bottom can be assessed using an indirect method. Energy reflection effects are demonstrated by 
comparing simulations of several impacts on samples with and without walls. The samples without walls are the same as the sample previously defined except that all walls are deleted at the beginning of the impact simulation. Although the particle's free fall is possible for the sample without walls, the impact simulation is also possible in this case because the impacting particle's incident velocity and the compression wave's speed magnitude are greater than the velocity of the soil particles (which is nil at the beginning of the simulation). Such simulations can evaluate the influence of the boundary conditions because the initial compression wave propagation occurs slightly in the same manner in both samples but the energy can obviously not be reflected on the walls if samples without walls are used.

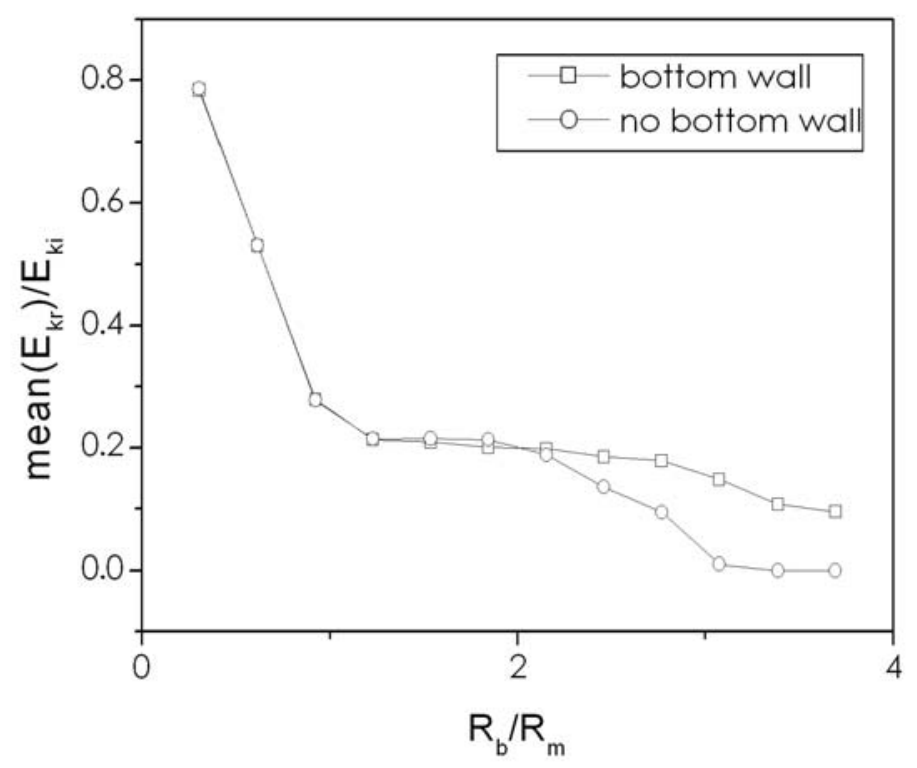

Figure 30. Changes in reflected energy with and without a bottom wall ( $V_{i}=20 \mathrm{~m} / \mathrm{s}, \alpha_{i}=45^{\circ}$,

$$
\left.\omega_{i}=0 \mathrm{rad} / \mathrm{s}, H / R_{b}=20\right) \text {. }
$$

Simulations show that for a given sample height, the variation in the impacting particle's kinetic energy at the end of the impact process $E_{k r}$ caused by removing the sample's walls largely depends on the $R_{b} / R_{m}$ parameter (Figure 30). For an impacting particle size such that $R_{b} / R_{m}<2$, removing the walls does not produce significant changes in the final mean impacting particle's kinetic energy $E_{k r}$. When the impacting particle size reaches larger values $\left(R_{b} / R_{m}>2\right)$, the final kinetic energy is not nil if the walls are preserved and vanishes otherwise. 
These results highlight that if the impacting particle size is small, the influence of energy reflection is negligible. On the other hand, the influence of energy reflection increases when the impacting particle enlarges. Energy reflection involves a second energy supply from the soil to the impacting particle since the impacting particle's reflected energy is greater if energy reflection on the walls is possible (Figure $31)$.

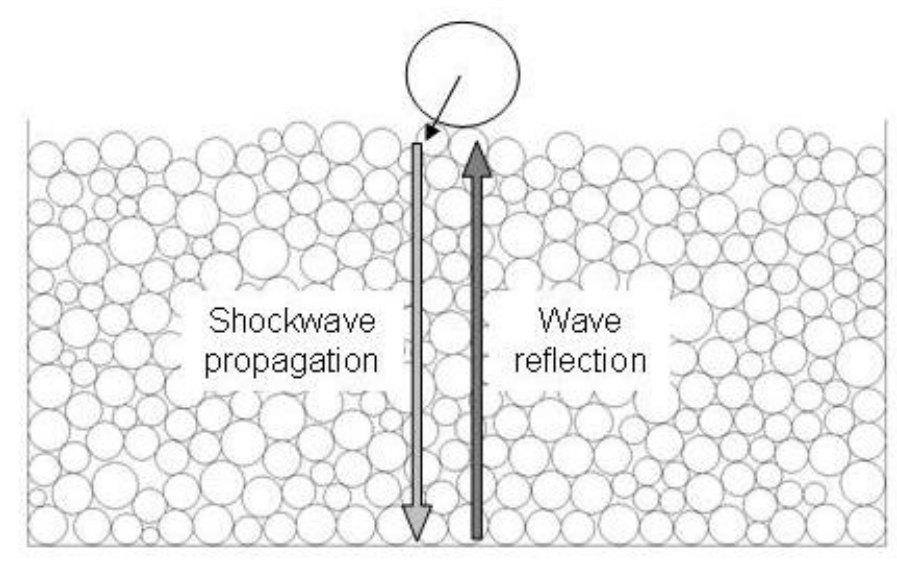

Figure 31. The shockwave's round trip.

The second energy transfer from the soil to the impacting particle is related to the arrival of the reflected shockwave after its round trip within the sample. Boundary effects are no longer negligible as soon as the compression wave is reflected on the sample walls and reaches the impact point again before the end of the impact.

For a small impacting particle size $\left(R_{b} / R_{m}<1\right)$, the second energy transfer does not occur because the impact duration is shorter than the shockwave's round trip within the sample. The impacting particle only bounces with the initial interaction between the impacting particle and a particle of the soil since, as shown in Figure 21, the impacting particle's normal velocity component inverses during the first phase. For $1<R_{b} / R_{m}<2$, removing the bottom wall does not produce significant effects: the existence of a strong phase makes it possible to develop arching effects within the medium such that energy reflection can occur. In addition, if the impacting particle's size is larger $\left(R_{b} / R_{m}>2\right)$, bouncing is mainly caused by the influence of the bedrock and, as a result, by energy reflected from the walls.

In conclusion, it appears that both the boundary conditions and the size of the impacting particle play a fundamental role in impacting particle bouncing. Analyzing the conditions in which bouncing occurs in detail is the purpose of the next section. 


\section{Does bouncing always occur?}

The previous results show that bouncing not only depends on the first energy exchange between the impacting particle and the soil, but it is also governed by shockwave propagation and reflection at the sample's boundaries. Incident conditions and physical processes inducing impacting particle bouncing are then explored in order to characterize different impact regimes and to specify their validity domain.

The impact process and bouncing are studied for varying impacting particle sizes, sample heights and impacting particle incident kinematic conditions. The influence of the incident rotational velocity $\omega_{i}$ will not be explored in this investigation to limit the number of parameters. The $\omega_{i}$ value is set at $0 \mathrm{rad} / \mathrm{s}$.

The range of other incident parameters is defined in accordance with real cases (Azzoni et al., 1991). The incident velocity $V_{i}$ domain is defined from $5 \mathrm{~m} / \mathrm{s}$ to $40 \mathrm{~m} / \mathrm{s}$ and incidence angle $\alpha_{i}$ ranges from $0^{\circ}$ to $75^{\circ}$. The ratio of the relative size of the impacting particle to the mean size of the soil particles $\left(R_{b} / R_{m}\right)$ varies between 0.3 and 15 to provide a wide range around the critical value $R_{b}=R_{m}$. The height of the sample $H$ ranges from $5 R_{m}$ to $25 R_{m}$.

For a given soil sample, the local soil particle layout variability is explored by doing several impact simulations for different impact points. Impact points are located far enough from lateral walls to prevent energy transfers due to shockwave reflection on lateral boundaries. As shown previously (Figure 10), a minimum of $N=100$ impact simulations on different impact points $d_{i}=0.05 \mathrm{~m}$ apart is required to ensure stable mean and standard deviation values for all calculated kinematic reflected quantities for a given set of parameters.

Since the impacting particle is placed exactly in contact with a soil particle, the coordinates of each impact point are precisely defined. Thus, the same impact points and, as a result, the same local configuration can be used for varying parameters. This procedure reduces the local effects because all simulations were run using the same local configurations.

A global definition of a bouncing occurrence is established in order to build a criterion that does not depend on local effects. The global bouncing criterion for a given set of parameters $\left(H, R_{b}, V_{i}, \alpha_{i}\right)$ is defined as the percentage of local bouncing occurrences for varying impact points and fixed incident conditions. As defined previously, the local bouncing occurrence criterion is the change in the impacting particle's normal velocity sign after the interaction with the soil. If the percentage of bouncing 
occurrences is higher than a threshold value $S$, the parameters tested are associated with a global bouncing occurrence. Otherwise, these parameters lead to the impacting particle stopping on the soil. In the following, the value $S=75 \%$ will be adopted. For example, for varying impacting particle radius $R_{b}$ values and fixed parameters $H, V_{i}$ and $\alpha_{i}$, the bouncing and stopping domains can be delimited as shown in Figure 32.

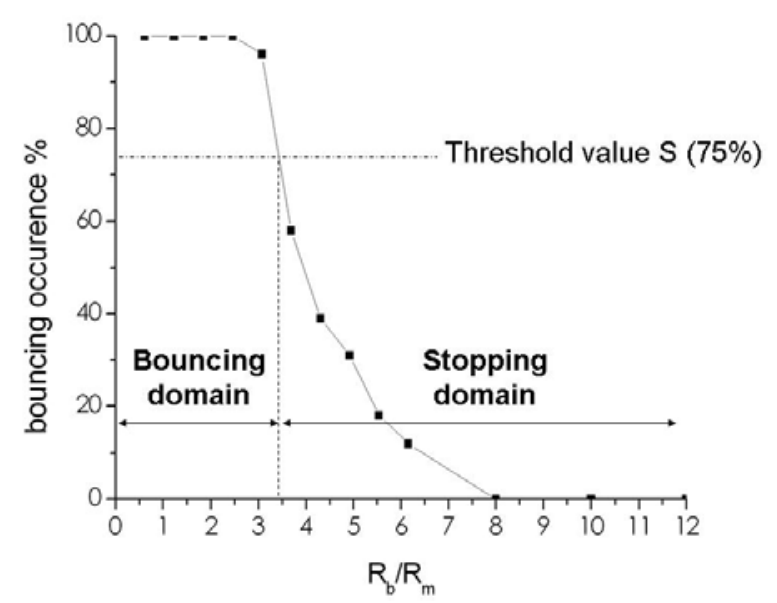

Figure 32. Definition of global bouncing occurrence for varying values of impacting particle radius $R_{b}$ and fixed values of $H, V_{i}$ and $\alpha_{i}$.

\subsection{Bouncing occurrence diagram}

The global bouncing occurrence criterion makes it possible to define sets of parameters $\left(H^{*}, R_{b}^{*}, V_{i}^{*}, \alpha_{i}^{*}\right)$ that induce the occurrence of impacting particle bouncing. For fixed incident kinematic conditions $\left(V_{i}^{*}, \alpha_{i}^{*}\right)$, the curve delimiting impacting particle bouncing and stopping occurrence is defined, in the two-dimensional space $\left(R_{b}, H\right)$, by the equation $H^{*}=f\left(R_{b}^{*}\right)$. Impacting particle bouncing is associated with the domain ' $A$ ' located below the curve. On the contrary, the total absorption of incident kinetic energy leading to the impacting particle stopping on the soil is associated with the domain ' $B$ ' above the curve. 


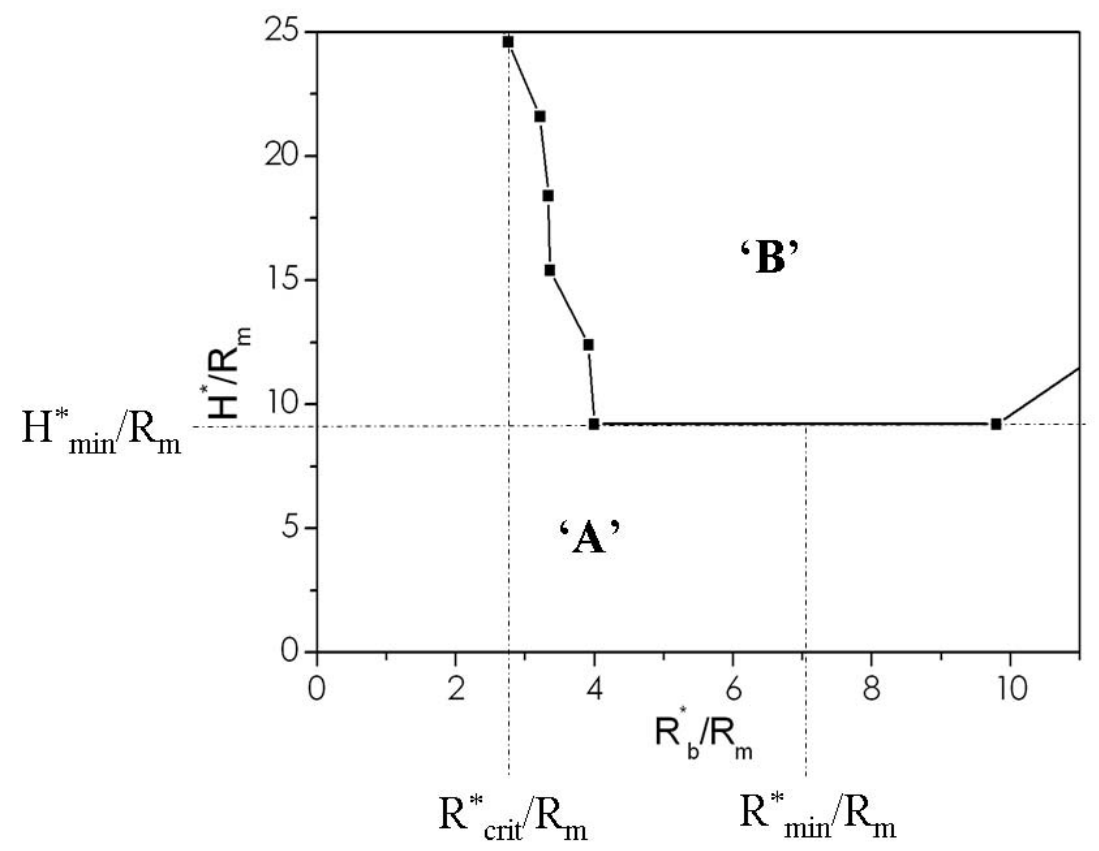

Figure 33. Bouncing occurrence diagram for fixed incident kinematic conditions.

As shown in Figure 33, the curve delimiting the ' $A$ ' domain and the ' $B$ ' domain in the $\left(R_{b}, H\right)$ plane passes through a minimum value corresponding to the pair of values $\left(R_{\min }^{*}, H_{\min }^{*}\right)$, and a vertical asymptote seems to exist for $R_{b}^{*} / R_{m} \approx R_{c r i t}^{*} / R_{m}$. If the depth of the granular medium is sufficiently small ( $H<H_{\min }^{*}$ ), bouncing always occurs. Moreover, if the impacting particle's radius is smaller than the asymptotic value $R_{c r i t}^{*}$, bouncing is possible whatever the sample height.

Three impact regimes can be defined depending on the impacting particle size based on the previous results. The first impact regime corresponds to the case where $R_{b}<R_{\text {crit }}^{*}$ (Figure 34). In the first impact regime, the impacting particle bounces in any incident condition and sample height. The second impact regime is delimited by an impacting particle radius range within the limits $R_{\text {crit }}^{*}<R_{b}<R_{\min }^{*}$ (Figure 34). In the second regime, the impacting particle does not bounce in all cases. The curve delimiting the ' $A$ ' domain and the ' $B$ ' domain in the $\left(R_{b}, H\right)$ plane decreases as $R_{b}^{*}$ values increase. The third impact regime has an impacting particle radius larger than $R_{\min }^{*}\left(R_{b}>R_{\min }^{*}\right.$; Figure 34). As for the second impact regime, the impacting particle cannot bounce for any $\left(R_{b}, H\right)$ pairs. Contrary to what is observed 
for the second regime, the delimiting curve is characterized by an increase in the $H^{*}$ value as the $R_{b}^{*}$ value increases.

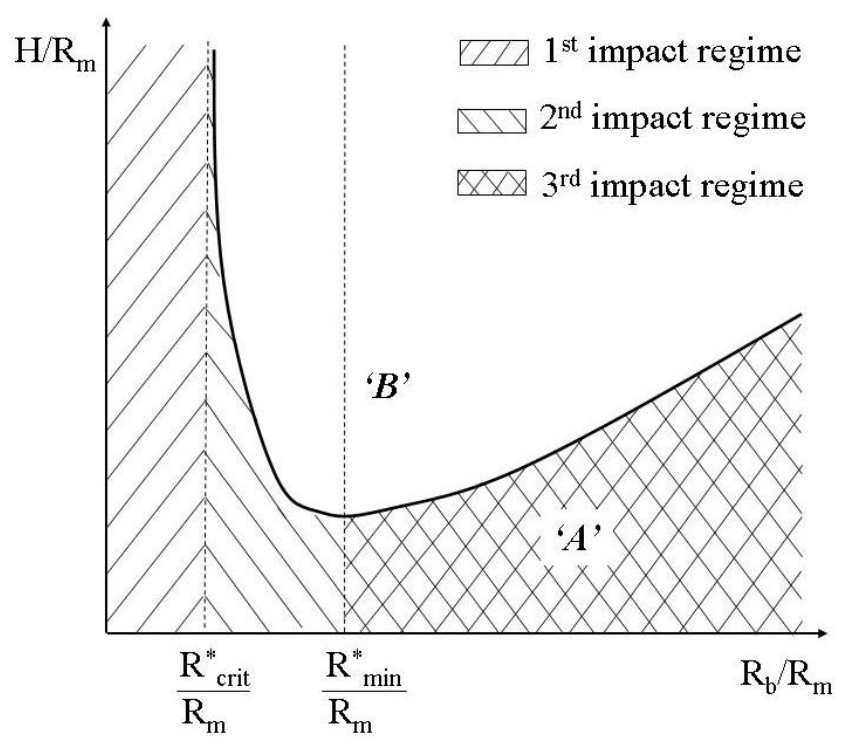

Figure 34. Definition of the impact regimes.

The asymptotical value $R_{\text {crit }}^{*}$ can be clarified by modelling the initial interaction as an impact between two rigid particles (the impacting particle and one soil particle) developed in the previous section of this paper. In the context of this model, Eq. (25) shows that for a small impacting particle radius ( $R_{b} / R_{m}<1$ ), which corresponds approximately to the first impact regime, the impacting particle's normal velocity sign changes during the first phase. In this context, bouncing directly occurs during the first phase according to the definition of local bouncing established previously. This means that a vertical asymptote exists because bouncing occurs during the first phase whatever the sample height if the impacting particle's radius is smaller than a critical value $\left(R_{b} / R_{m}<1\right)$.

On the other hand, for the second and the third impact regimes, the initial interaction phase between the block and the soil does not directly lead to the impacting particle bouncing and the impacting particle continues penetrating the soil after the first phase. The impacting particle's incident kinetic energy is totally transferred to the soil by successive interactions between the impacting particle and the soil particles until it reaches zero. These interactions lead to the propagation of successive shock waves within the sample. As the total incident kinetic energy of the impacting particle is transferred to the soil, bouncing is only possible if energy is transferred again from the soil particles to the impacting particle. 
This second energy exchange occurs if the incident kinetic energy initially transferred is not totally dissipated during the shockwave's round trip.

Simulations demonstrate that the impacting particle's incident kinetic energy is mainly transferred to the soil during the first interaction between the block and the soil. Then, in a first approximation, the total energy transfer phenomenon can be modelled by the propagation of the first shockwave through the sample (Figure 35). The wave's initial energy is therefore nearly equal to the impacting particle's incident kinetic energy.

If the incident energy is totally transferred to the soil, i.e. for $\left.R_{b} / R_{m} \in\right] 1, \infty[$, the curve limiting the ' $A$ ' domain and the ' $B$ ' domain in the two-dimensional space $\left(R_{b}, H\right)$ corresponds to equality between the incident impacting particle's kinetic energy $E_{k i}$ and the total dissipated energy during the first shockwave's round trip $E_{\text {diss }}$. The impacting particle's incident kinetic energy $E_{k i}$ is directly related to the impacting particle's size by the relation $E_{k i}=a R_{b}^{2}$ where $a=\frac{\pi}{2} \rho_{b} V_{i}^{2}$ is a constant value over the set of simulations considered.

During shockwave propagation, the energy propagates along contact chains and is dissipated at contact points. The total dissipated energy during the shockwave's round trip is then related to the number of loaded contacts. A qualitative analysis of simulations emphasizes that the propagation process activates contact chains located within a conical volume behind the impacting particle. The number of loaded contacts depends on the area of this conical volume. This area is estimated based on simple geometrical considerations.

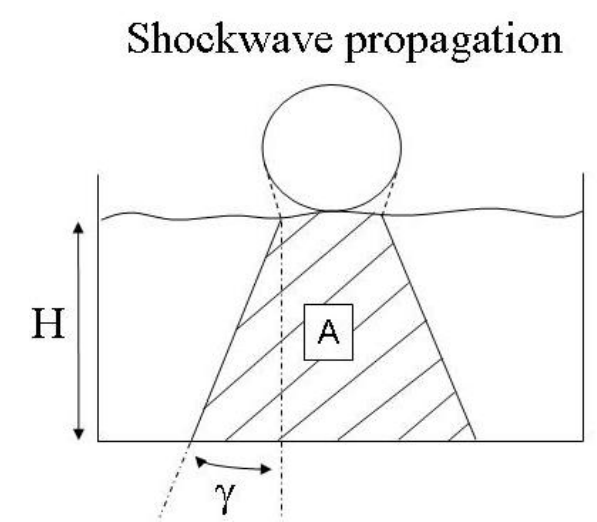

\section{Shockwave reflection}

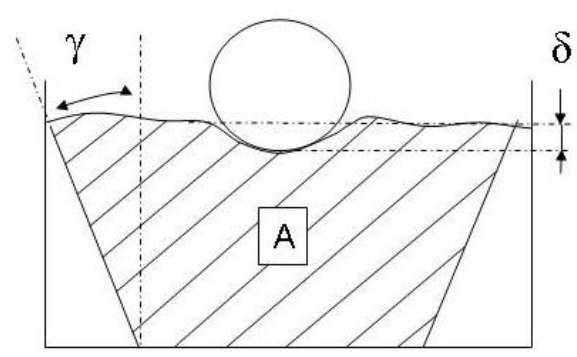

Figure 35.Conical volume associated with loaded contact chains 


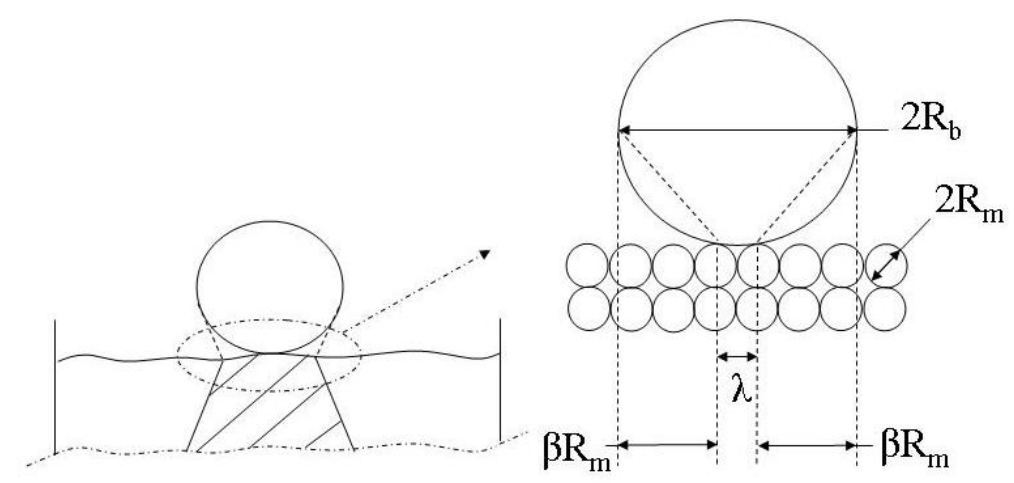

Figure 36. Geometrical characterization of the loaded surface $\lambda$.
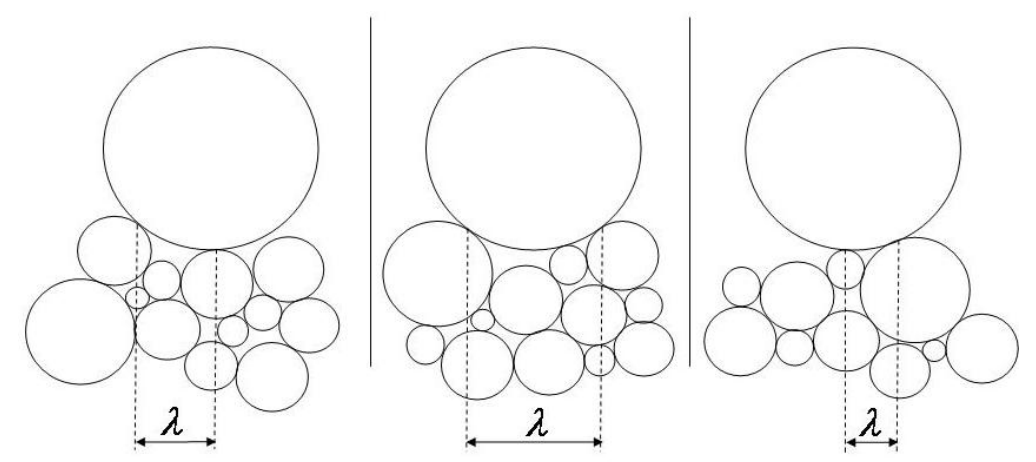

Figure 37. Influence of the local soil particles' geometrical configuration on the $\lambda$ parameter.

As shown in Figure 36, the loaded surface $\lambda$ is the surface that includes all the potential contact points between the impacting particle and the soil's surface. The loaded surface $\lambda$ depends to a great extent on the impacting particle's size and the local soil particles' geometrical configuration (Figure 37). The simplest relation translating geometrical effects is: $\lambda=2\left(R_{b}-\beta R_{m}\right)$. The parameter $\beta$ ranges within $\left[0, \frac{R_{b}}{R_{m}}\right]$ and accounts for the variability of the local particles' geometrical configuration (Figure 37).

Loaded particles during shockwave propagation and reflexion are located within a truncated cone. The height of this cone is $2 H-\delta$ since the shockwave covers a distance $H$ on its way from the impact point to the sample's lower boundary and a distance $H-\delta$ on its way back to the soil surface (Figure 35), where $\delta$ denotes the maximum penetration of the impacting particle within the soil. Moreover, the upper surface of the cone is equal to $\lambda$ and its angle is set at the $\gamma$ value.

The area $A$ (Figure 36) of the truncated cone is then: 


$$
A=2(2 H-\delta)\left(R_{b}-\beta R_{m}\right)+(2 H-\delta)^{2} \tan \gamma
$$

Establishing a relevant expression of the energy dissipated on a contact depending on the contact location and the contact force is not straightforward. However, since the purpose of the study is to define a simple model relevant for first-order phenomena, the dissipated energy is assumed, in a first approximation, to be the same on each contact. Total dissipated energy is therefore only proportional to the number of activated contacts. It is then directly proportional to the area $A$. Dissipated energy $E_{\text {diss }}$ can then be written as $E_{\text {diss }}=b\left[2(2 H-\delta)\left(R_{b}-\beta R_{m}\right)+(2 H-\delta)^{2} \tan \gamma\right]$ where $b$ is a constant parameter.

The equality of dissipated and incident energies leads to the equation:

$$
(2 H-\delta)^{2} b \tan \gamma+(2 H-\delta) 2 b\left(R_{b}-\beta R_{m}\right)-a R_{b}^{2}=0
$$

Given that the loaded surface $\lambda$ is positive, $R_{b}-\beta R_{m}$ is also a positive value. Assuming that $\beta$ is a constant value, the validity domain of Eq. (30) is then $\left.\frac{R_{b}}{R_{m}} \in\right] \beta, \infty[$. The positive solution of Eq. (30) to the variable $H$ is:

$$
\frac{H^{*}}{R_{m}}=\frac{\delta^{*}}{2 R_{m}}+\frac{1}{2 \tan \gamma}\left(\frac{R_{b}^{*}}{R_{m}}-\beta\right)\left(\sqrt{1+\frac{a \tan \gamma}{b} \frac{\left(\frac{R_{b}^{*}}{R_{m}}\right)^{2}}{\left(\frac{R_{b}^{*}}{R_{m}}-\beta\right)^{2}}}-1\right)
$$

Thus, $\frac{H^{*}}{R_{m}}=f\left(\frac{R_{b}^{*}}{R_{m}}\right)$ with $f(x)=\frac{\delta^{*}}{2 R_{m}}+\frac{1}{2 \tan \gamma}(x-\beta)\left(\sqrt{1+\frac{a \tan \gamma}{b} \frac{x^{2}}{(x-\beta)^{2}}}-1\right)$.

In the case of $\frac{R_{b}^{*}}{R_{m}} \rightarrow \beta$, the solution of Eq. (31) tends toward a finite value.

$$
\frac{H^{*}}{R_{m}}=\frac{1}{2} \sqrt{\frac{a}{b \tan \gamma}} \beta+\frac{\delta^{*}}{2 R_{m}}
$$

With $\frac{R_{b}^{*}}{R_{m}}>>\beta$, a power series expansion leads to the reduced expression of the $f$ function:

$$
\frac{H^{*}}{R_{m}}=\frac{1}{4} \sqrt{\frac{a}{b \tan \gamma}} \frac{R_{b}^{*}}{R_{m}}+\frac{\delta^{*}}{2 R_{m}}
$$




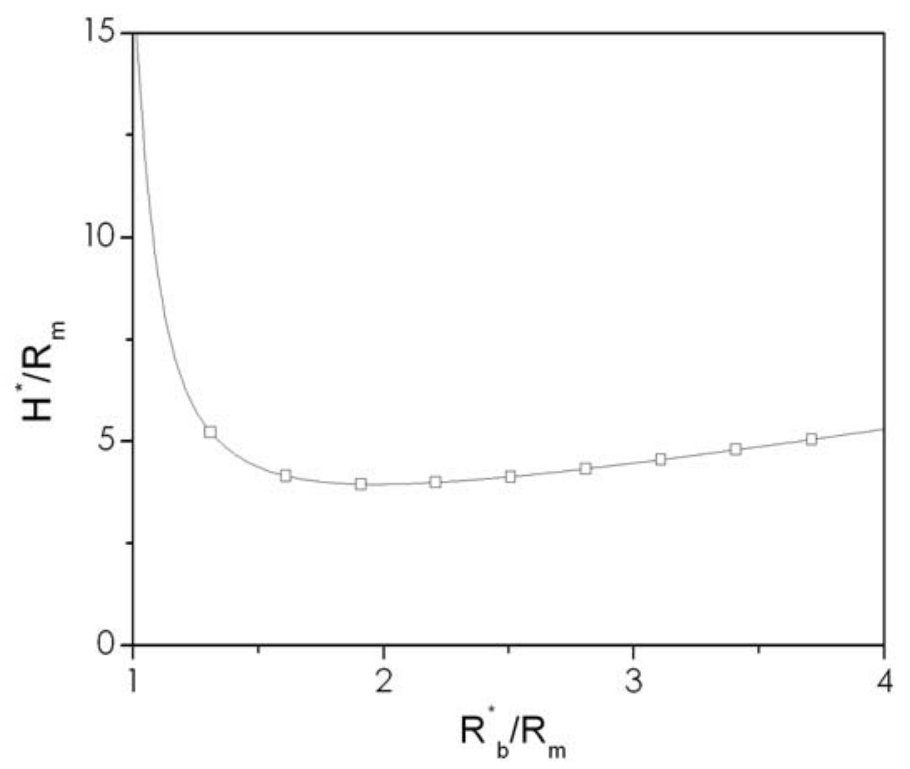

Figure 38. Variation of $f$ function $\left(a=2 e 5, b=5 e 4, \beta=1, \gamma=0.2^{\circ}\right)$.

As shown in Figure 38, the curve giving the variation of $f$ with $\left.\frac{R_{b}^{*}}{R_{m}} \in\right] \beta, \infty[$ is very similar to that shown in Figure 33.

In particular, an oblique asymptote exists for $\frac{R_{b}^{*}}{R_{m}} \rightarrow \infty$, and the curve passes through a minimum value. Although a vertical asymptote does not exist in all cases, the limit value shown in Eq. (32) for $\frac{R_{b}^{*}}{R_{m}} \rightarrow \beta$ depends to a large extent on the $\gamma$ parameter and tends towards infinite for small values of $\gamma$. Therefore, if the shockwave propagation is unidirectional $(\gamma \rightarrow 0)$, a vertical asymptote exists.

The influence of each parameter of the theoretical model on the curve giving the variation of function $f$ is studied. The increase in parameter $a$ related to the incident kinetic energy leads to an increase in the limit sample height $H^{*}$ for any impacting particle size (Figure 39). On the contrary, the increase in parameter $b$ related to dissipated energy induces a decrease in the limit sample height $H^{*}$, whatever the impacting particle size (Figure 40). Since parameters $\beta$ and $\gamma$ are also related to dissipated energy, the increase in these parameters also leads to a decrease in $H^{*}$ (Figure 41; Figure 42). 
Finally, it can be noted that both parameters $\beta$ and $\gamma$ mainly influence function $f$ in the second impact regime and the values of the transitions between impact regimes, whereas parameters $a$ and $b$ mainly influence the limiting curve on the third impact regime. These results show that the second impact regime is mainly governed by the shape of the volume that contains loaded contacts, whereas the third regime is highly dependent on the relative influence of the incident energy on the dissipative capabilities of the soil.

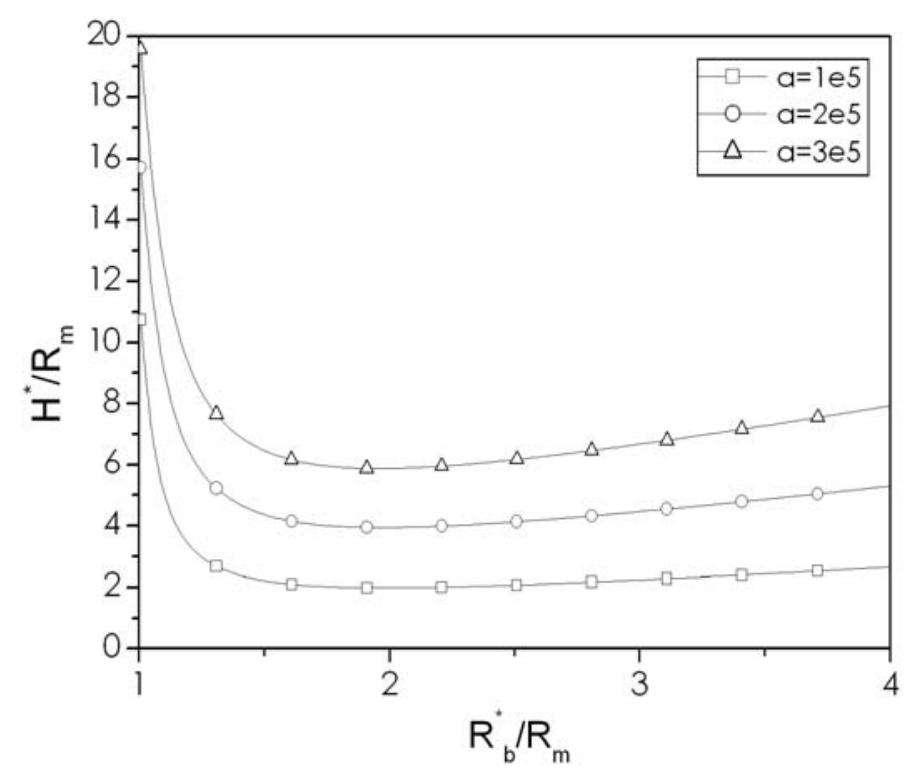

Figure 39. Variation of function $f$ for different a values $\left(b=5 e 4, \beta=1, \gamma=0.2^{\circ}\right)$.

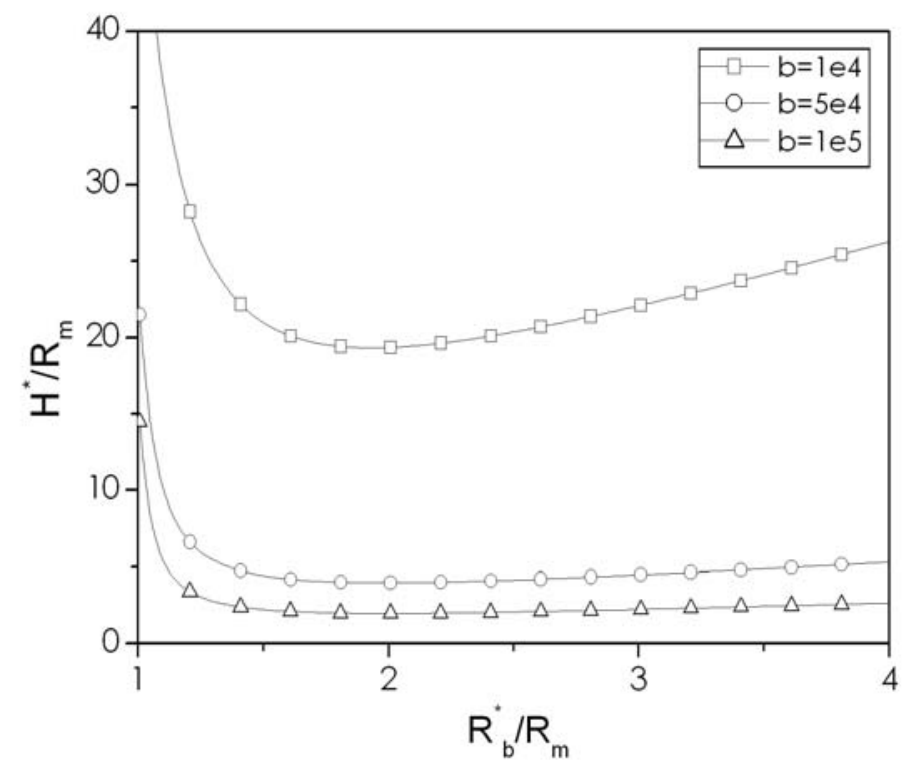

Figure 40. Variation of function $f$ for different $b$ values $\left(a=2 e 5, \beta=1, \gamma=0.2^{\circ}\right)$. 


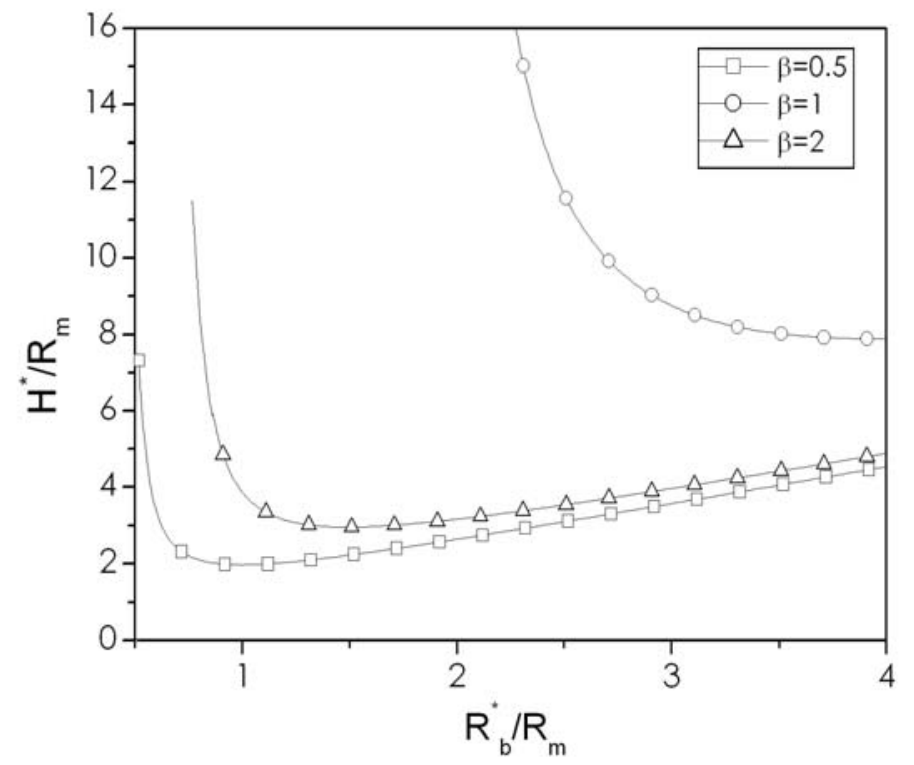

Figure 41. Variation of function $f$ for different $\beta$ values $\left(a=2 e 5, b=5 e 4, \gamma=0.2^{\circ}\right)$.

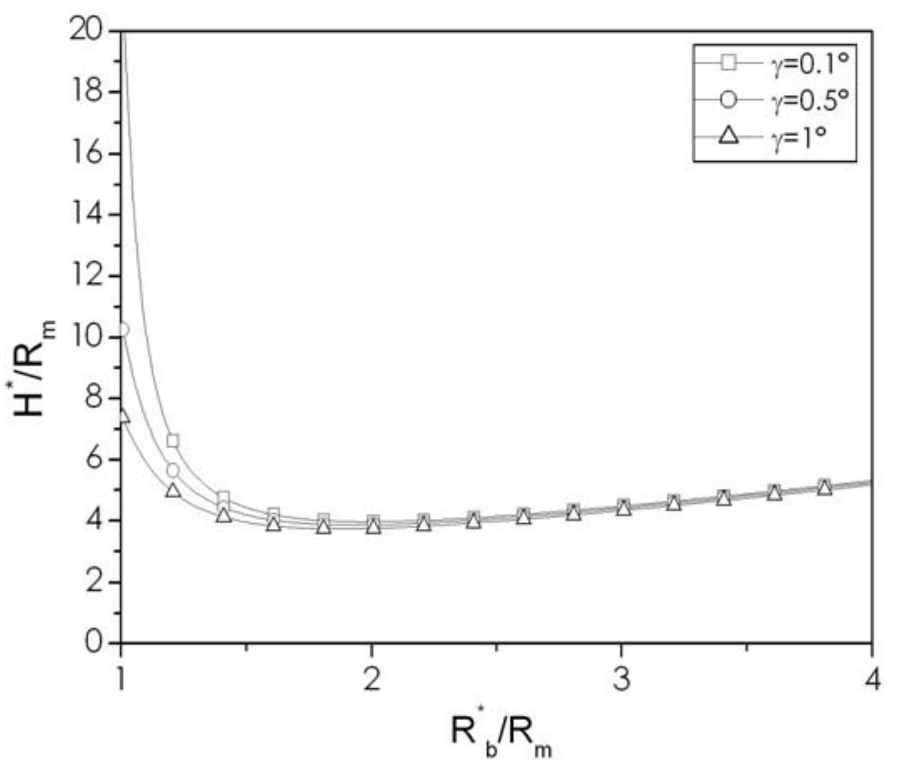

Figure 42. Variation of function $f$ for different $\gamma$ values $(a=2 e 5, b=5 e 4, \beta=1)$.

On the third impact regime, impacting particle penetration becomes appreciable (Figure 44). Since impacting particle size is high, the incident kinetic energy is high and the dissipation processes over the round trip of the shockwave are not sufficient to dissipate the incident kinetic energy. Substantial 
impacting particle penetration (Figure 44) occurs because the kinetic energy that is not dissipated leads to substantially deforming the sample.

Interestingly, as observed in Figure 44, the penetration depth reaches an asymptotic value $\delta_{\text {lim }}$ when the size of the impacting particle increases. Thus, bouncing occurs when the height $h_{c}$ (Figure 43) of the bottom layer between the bottom wall and the impacting particle is equal to a critical value, which is a function of the sample height. For the sample considered $\left(H^{*} / R_{m}=12.3\right.$ ), the penetration depth reaches an asymptotic value $\delta_{\lim } / R_{m}=8.5$ and the height of the bottom layer is equal to $h_{c}=H^{*}-\delta_{\lim }=3.8 R_{m}$. The compacity of the bottom layer is then high enough to ensure the growth of stable (nondissipative) force chains in which normal forces can develop and contribute to the bouncing of the impacting particle.

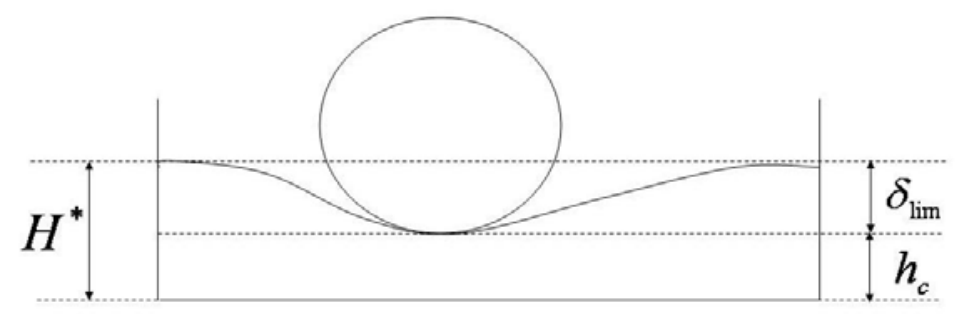

Figure 43. Relation between sample height $H^{*}$, penetration depth $\delta^{*}$ and bottom layer height $h_{c}$.

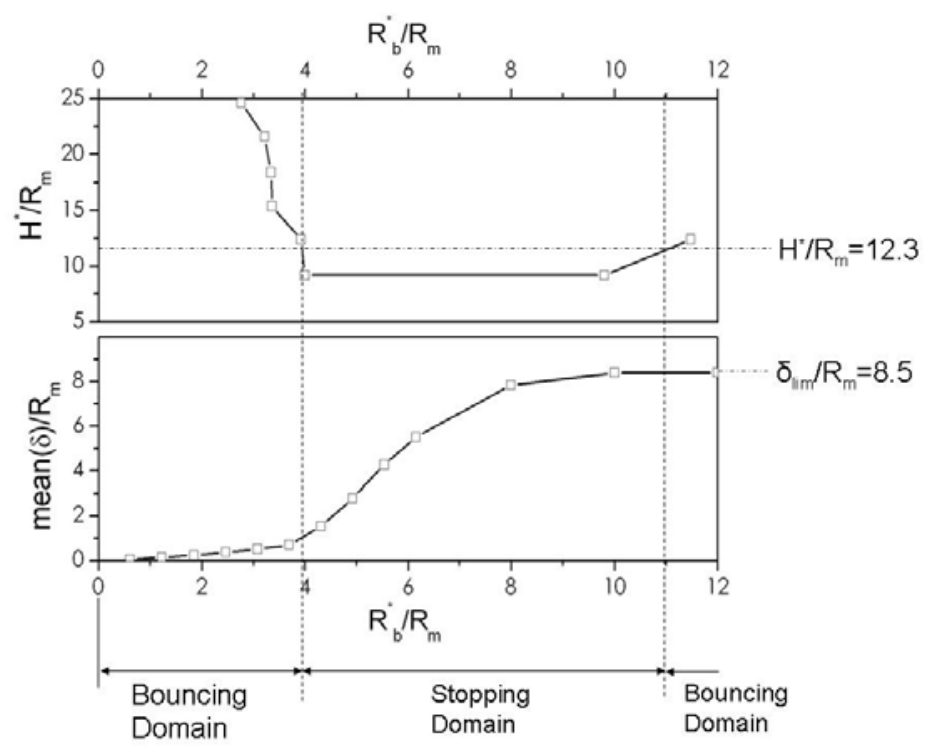

Figure 44. Impacting particle penetration for varying values of $R_{b}\left(V_{i}=20 \mathrm{~m} / \mathrm{s}\right.$,

$$
\alpha_{i}=45^{\circ}, H / R_{m}=12.3 \text { ). }
$$




\subsection{Influence of incident velocity and particle shape on the bouncing occurrence diagram's}

properties

Given that a theoretical model taking into account all these phenomena would be very complex to develop, a parametrical study is necessary to determine the influence of the incident parameters. The occurrence of bouncing, which depends on incident impacting particle velocity $V_{i}$ and particle shape, was therefore explored.

The simulations show that when the incident kinetic energy is high, i.e. when the impacting particle is very large compared to soil particles, contact forces and interpenetrations reach very high values, bringing the simulation to the limits of DEM. This is the reason why the developments considered in the following are only based on the first two regimes $\left(R_{b}^{*} / R_{m}<6\right)$.

The value of the $R_{c r i t}^{*}$ parameter depends to a large extent on the incident velocities $V_{i}$. The numerical results show that $R_{c r i t}^{*}$ increases for increasing incident velocities $V_{i}$ (Figure 45). On the contrary, the incident velocity's influence on the $\left(R_{\min }^{*}, H_{\text {min }}^{*}\right)$ minimum seems to be reduced as regards the precision of the simulations (Figure 46). The influence of the incident velocity $V_{i}$ on $R_{c r i t}^{*}$ can be approached by relating the incident velocity to the contact force during the first interaction between the impacting particle and the soil. Indeed, as the $R_{c r i t}^{*}$ parameter is the upper limit of the first impact regime, $R_{c r i t}^{*}$ is the size of the largest particle that can directly bounce during the first phase. For the Hertz-Mindlin model used in the simulations, the influence of the kinematic parameters on the contact force between the interacting particles is taken into account. According to the Hertz-Mindlin model, the maximum normal interaction force $F_{n \text {,max }}$ during the interaction is related to the normal component of the incident velocity $V_{n, I}$ (Wolf, 1996):

$F_{n, \max } \propto K_{n}^{2 / 5} R_{b}^{3 / 5} V_{n, i}^{6 / 5}$

Eq. (34) shows that during the first interaction between the impacting particle and the soil, the normal maximum force varies in the same way as the normal component of the incident velocity $V_{n, I}$. Noting that $V_{i}$ and $V_{n, I}$ are related by the local incidence angle $\alpha_{l}, F_{n, \max }$ and $V_{i}$ also vary in the same way. As 
a result, for increasing incident velocity, the contact force increase during the initial interaction phase induces an increase in $R_{c r i t}^{*}$. This property is in agreement with the numerical results shown in Figure 45. The influence of particle shape must also be considered. Since for the same size distribution, the porosity of the clump particle assembly is higher than the porosity of the spherical particle assembly, soil type B is more compact than soil type A. There are consequently fewer possibilities for particle rearrangement for soil type B than for soil type A. Therefore, dissipated energy is less for clump assemblies, which is confirmed by the fact that the $H_{\min }^{*}$ value is higher for soil type B (Figure 47). On the contrary, $R_{c r i t}^{*}$ values are approximately constant for all particle shapes (Figure 47). As shown in Eq. (24), the $R_{c r i t}^{*}$ value is essentially determined by the ratio of the mass of impacting particles to the mass of impacted soil particles. As a consequence, $R_{\text {crit }}^{*}$ does not vary for varying particle shapes because the particle sizes are the same for soil type A and soil type B.

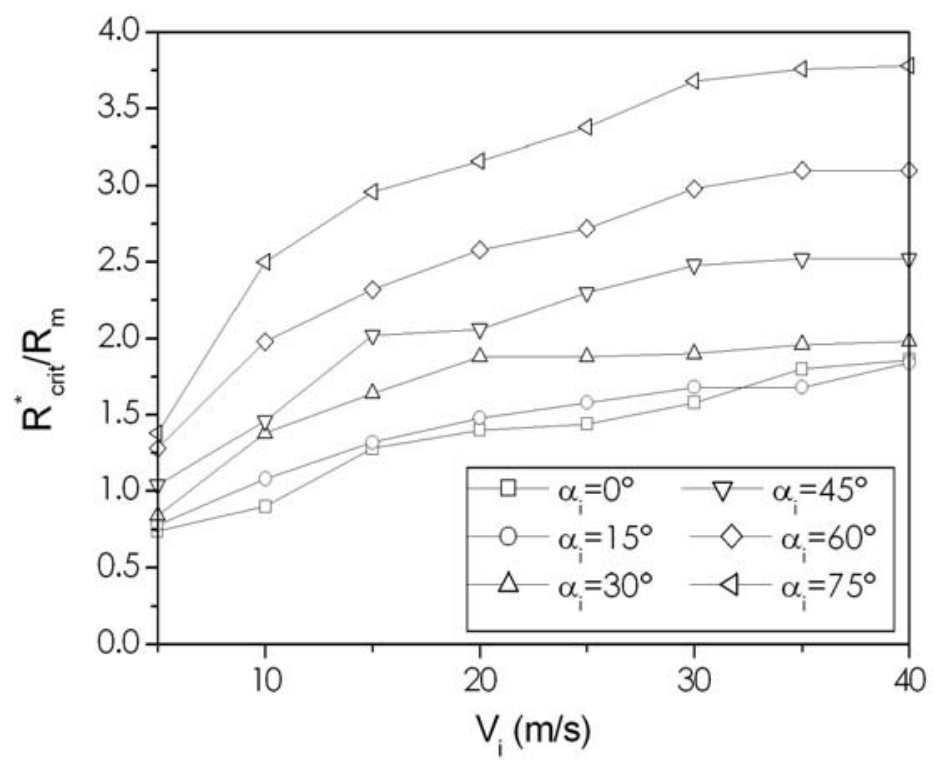

Figure 45. $R_{\text {crit }}^{*}$ as a function of the incident velocity $V_{i}$ for different incidence angles $\alpha_{i}$. 


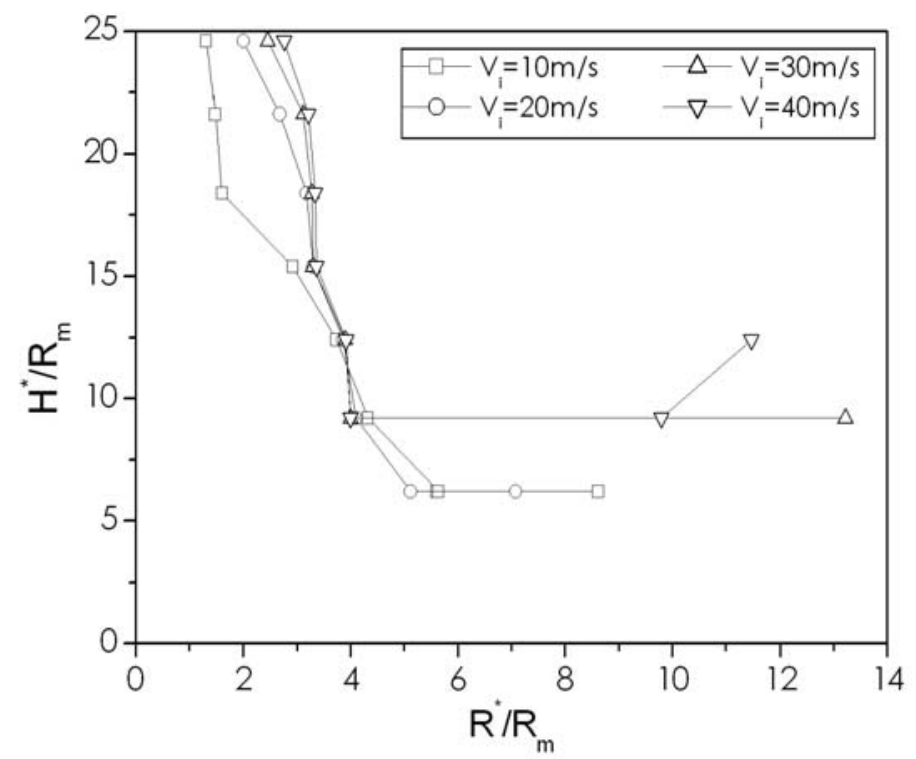

Figure 46. Bouncing occurrence compared to $V_{i}\left(\alpha_{i}=45^{\circ}\right)$.

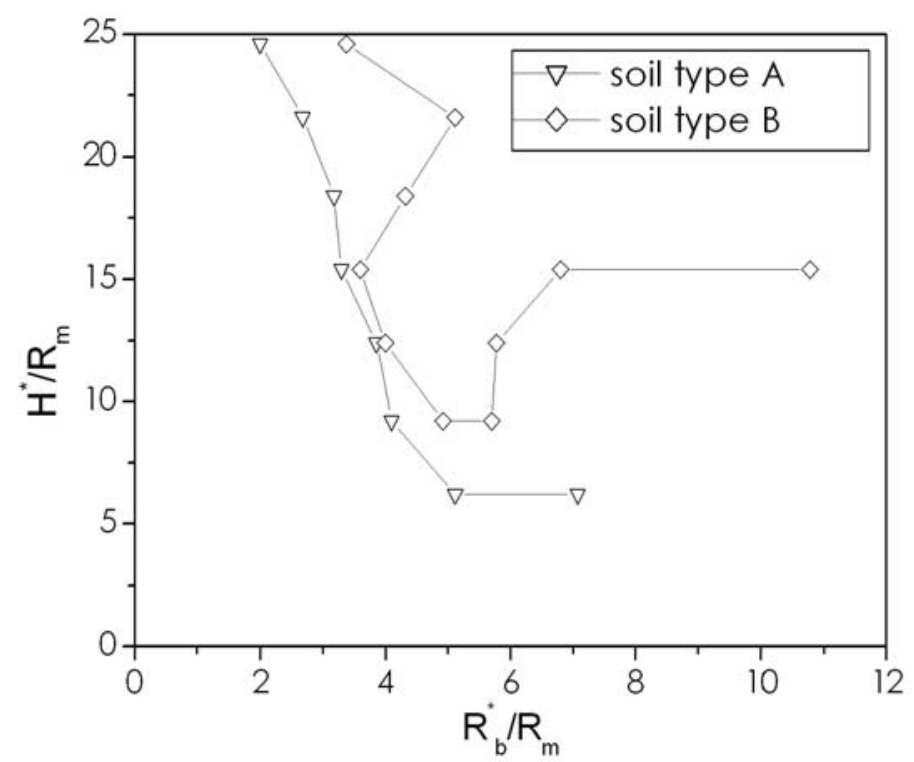

Figure 47. Bouncing occurrence diagram for sphere assemblies and clump assemblies $\left(V_{i}=20 \mathrm{~m} / \mathrm{s}\right.$, $\left.\alpha_{i}=45^{\circ}\right)$.

\section{Conclusion}

The numerical investigations presented in this paper provide an overview of the phenomena governing the impact of a block on a coarse granular soil. 
The soil is described as a noncohesive granular medium using the discrete element method. Extensive numerical simulations were then performed for different impacting particle and medium properties. These simulations brought out the phenomena involved in energy exchanges between the impacting particle and the granular medium.

The results first show that the interaction between the impacting particle and the soil particles can be divided into three successive phases: the initial energy exchange from the impacting particle to the soil, the propagation of a shockwave within the sample and a second energy exchange from the soil particles to the impacting particle if the reflected wave reaches the soil surface before the end of the impact. The results also highlight the role of the local grain's spatial layout, the impacting particle size, the incident kinematic parameters and the boundary conditions on the different impact phases.

Based on these physical investigations, whether bouncing occurs can be explored for given soil properties. Bouncing is defined for all local configurations by exploring several impact points and defining a global bouncing existence criterion. This procedure provides a definition of a diagram delimiting impacting particle bouncing and stopping for all local configurations depending on the sample height, impacting particle size and incident kinematic conditions.

For given incident kinematic conditions, this diagram illustrates the three impact regimes depending on impacting particle size. The first impact regime corresponds to small impacting particle size ( $\left.R_{b} / R_{m} \leq 1\right)$. In this case, the impact process is mainly governed by the initial interaction between the impacting particle and the soil. The second impact regime is associated with an intermediate impacting particle radius $\left(1<R_{b} / R_{m} \leq 6\right)$. For the second regime, impacting particle bouncing is attributable to a second energy supply from the soil after the shockwave's round trip through the sample. The second energy supply is possible if the impacting particle's incident energy is not fully dissipated through frictional processes during the shockwave's round trip. The third impact regime corresponds to a large impacting particle size $\left(R_{b} / R_{m}>6\right)$. The impact of the impacting particle here leads to substantial impacting particle penetrations within the sample and bouncing is the result of the interaction of the impacting particle with a thin compacted layer.

Although the results obtained clearly show that three impact regimes exist in the context of the numerical simulations performed, whether three impact regimes exist for real rockfall events has not yet been established conclusively. The first limitation to the existence of all three regimes is the difference 
between 2D simulations and 3D reality. Indeed, in a 3D context, since particle rearrangements can occur more easily, the limits of the regimes are certainly not the same. In particular, one could assume that the domain corresponding to the second impact regime could be highly restricted because this regime is mainly caused by energy transfers associated with elastic energy propagation and small-particle rearrangements. The 3D context could therefore encourage the direct transition from the first regime to the third regime. In addition, for large boulder sizes, energy transfers occurring during real impacts of rocks on scree slopes may also be different because contact attrition, crack propagation and rock breakage may occur. However, the results obtained in this study provide a basis for further simulation campaigns in which energy dissipation processes would be modelled more precisely.

Finally, the existence of several impact types means that general impact models that can be used by practitioners for all impact regimes need to be developed. For this purpose, the approach presented in this paper has been used to develop a general stochastic framework (Bourrier et al., 2007) leading to the definition of a stochastic impact model valid for any impact regime.

\section{References}

Azzoni, A.; Rossi P.P.; Drigo E. ; Giani G.P.; Zaninetti A. 1991. In situ observations of rockfalls analysis parameters. In Landslides. Rotterdam: Balkema (Bell ed.): 307-314.

Bagi, K. 2005. An algorithm to generate random dense arrangements for discrete element simulations of granular assemblies. Granular matter 7: 31-43.

Bayes, T. 1763. Essay towards solving a problem in the doctrine of chances. Philosophical Transactions of the Royal Society of London 53: 370-418; 54: 296-325.

Beladjine, D. 2007. Etude expérimentale du processus de collision d'une bille sur un empilement granulaire: application au mécanisme de saltation dans le transport éolien. PhD Thesis,Univ.Rennes 1. Beladjine, D.; Ammi, M.; Oger, L.; Valance, A. 2007. Collision between an incident bead and a threedimensional granular packing. Physical review E 75, 061305: 1-12.

Beladjine, D.; Valance, A.; Ammi, M.; Oger, L. 2005. Experimental and numerical study of the collision between an incident bead and a three dimensional granular packing. Proc. Int. Cong. Powders and Grains (Stuttgart, Germany): 1207-1210.

Bertrand, D.; Nicot, F.; Gotteland, P.; Lambert, S.; Derache, F. 2006. Modelling a geo-composite cell using discrete analysis. Computer \& geotechnics 32(8): 564-577. 
Bourrier, F.; Nicot, F.; Darve, F. 2007. Rockfall modelling: Numerical simulation of the impact of a particle on a coarse granular medium. Proc. $10^{\text {th }}$ Int. Congr. on NUmerical MOdel in Geomechanics (Rhodes, Greece): 699-705.

Bozzolo, D.; Pamini, R.; Hutter, K. 1988. Rockfall analysis - a mathematical model and its test with field data. Proc. 5th Int. Symposium on Landslides (Lausanne, Switzerland) 1: 555-563.

Campbell, C.S. 2003. A problem related to the stability of force chains. Granular Matter 5: 129-134. Ciamarra, M.P.; Lara, A.H.; Lee, A.T.; Goldman, D.I.; Vishik, I.; Swinney, H.L. 2004. Dynamics of drag and force distributions for projectile impact in a granular medium. Physical Review Letters 92, 194301: 14.

Coste, C.; Falcon, E.; Fauve, S. 1997. Solitary waves in a chain of beads under Hertz contact. Physical Review E. 56(5): 6104-6117.

Crassous, J.; Beladjine, D.; Valance, A. 2007. Impact of a projectile on a granular medium described by a collision model. Physical Review Letters 99 (24): 248001.

Cundall, P.A. \& Strack, O.D.L. 1979. A discrete numerical model for granular assemblies. Geotechnique 29: 47-65.

Cundall, P.A. 1988. Computer simulations of dense spheres assemblies. In Micromechanics of Granular Materials. Amsterdam: Elsevier Science Publisher B.V. (M. Satake \& J.T. Jenkins eds.): 113-123.

Deluzarche, R. \& Cambou, B. 2006. Discrete numerical modelling of rockfill dams. Int. J. Numer. Anal. Meth. Geomech. 30: 1075-1096

Descoeudres, F.; Zimmermann, T. 1987. Three-dimensional dynamic calculation of rockfalls. Proc. 6th Int. Congress of Rock Mechanics (Montreal, Canada): 337-342.

Dorren, L.K.A. 2003. A review of rockfall mechanics and modelling approahces. Progress in Physical Geography 27(1): 69-87.

Falcetta, J. L. 1985. Un nouveau modèle de calcul de trajectoires de blocs rocheux. Revue Francaise de Geotechnique 30: 11-17.

Frémond, M. 1995. Rigid bodies collisions. Physics Letters A 204: 33-41.

Guzzetti, F.; Crosta, G.; Detti, R.; Agliardi F. 2002. STONE: a computer program for the three dimensional simulation of rock-falls. Computer \& Geosciences 28: 1079-1093.

Gilks, W.R.; Richardson, S.; Spiegelhalter, D.J. 2001. Markov Chain Monte Carlo in Practice. NewYork: Chapman \& Hall. 
Goldsmith, W. 1960. Impact: the theory and physical behaviour of colliding solids. Doved Ed.

Goodman, R.E. 1980. Introduction to rocks mechanics. Boston: PWS Publishing Company.

Hostler, S.R. \& Brennen C.E. 2005. Pressure wave propagation in granular bed. Physical review E 72(3)

031303: 1-13.

Itasca Consulting Group. 1999. PFC2D Theory and background. Minneapolis: Itasca.

Itasca Consulting Group. 1999. PFC2D User’s manual. Minneapolis: Itasca.

Job, S.; Melo, F.; Sokolow, A.; Sen, S. 2007. Solitary wave trains in granular chains: experiments, theory and simulations. Granular Matter 10: 13-20.

Kirkby, M.J. \& Statham I. 1975. Surface movement and scree formation. Journal of geology 83: 349-362. Kobayashi, Y.; Harp, E. L.; and Kagawa, T. 1990. Simulation of rockfalls triggered by earthquakes. Rock Mech. Rock Eng. 23: 1-20.

Laouafa, S. \& Nicot, F. 2003. Modélisation numérique de l'impact d'un bloc rocheux sur un sol composé d'éboulis. Revue Française de Géotechnique 109: 87-97.

Mindlin, R.D. \& Deresiewicz, H. 1953. Elastic spheres in contact under varying oblique forces. Journal of Applied Mechanics 20: 327-344.

Oger L., Ammi M., Valance A., Beladjine D. 2005. Discrete Element Method to study the collision of one rapid sphere on 2D and 3D packings. Eur. Phys. J. E 17: 467-476.

Nishida M., Tanaka K., Matsumoto Y. 2004. Discrete Element Method simulation of the restitutive characteristics of a steel spherical impacting particle from a particulate aggregation. JSME International journal 47 (3): 438-447.

Pfeiffer, T. \& Bowen, T. 1989. Computer simulation of rockfalls. Bull. Ass. Eng. Geol. 26 (1): 135-146. Radjai, F.; Wolf, D.E.; Jean, M.; Moreau, J.J. 1998. Bimodal character of stress transmission in granular packings. Physical Review Letters 80 (1): 61-64.

Rioual, F.; Valance, A.; Bideau, D. 2000. Experimental study of the collision process of a grain on a twodimensional granular bed. Physical Review E. 62: 2450-2459.

Rioual, F.; Valance, A.; Bideau, D. 2003. Collision process of a bead on a two-dimensional packing: Importance of the inter-granular contacts. Europhys. Lett. 61(2): 194-200

Sadd, M.H.; Adhikari, G.; Cardoso, F. 2000. DEM simulation of wave propagation in granular materials. Powder technology 109: 222-233. 
Somfai, E.; Roux, J.N.; Snoeijer, J.H.; Van Hecke, M.; Van Saarloos, W. 2006. Elastic wave propagation in confined granular systems. Physical Review E. 72 021301: 1-18.

Stronge, W. J. 2000. Impacts mechanics. Cambridge: Cambridge University Press.

Tanaka K., Nishida M., Kunimochi T., Takagi T. 2002. Discrete element simulation and experiment for dynamic response of two-dimensional granular matter to the impact of a spherical impacting particle. Powder Technology 124: 160-173.

Thornton C., Ning Z. 1998. A theoretical model for the stick/bounce behaviour of adhesive, elastic-plastic spheres. Powder technology 99:154-162.

Toiya, M.; Hettinga, J.; Losert, W. 2007. 3D imaging of particle motion during penetrometer testing. From microscopic to macroscopic soil mechanics. Granular Matter 9: 323-329.

Wolf, D.E. 1996. Modelling and computer simulation of granular media. In Computational Physics. Heidelberg: Springer (Hoffmann,K.H.; Schreiber, M. eds.). 